\title{
Convergence Results on the Boundary Conditions for 2D Large-Scale Primitive Equations in Oceanic Dynamics
}

\author{
Yuanfei Li (iD \\ School of Mathematics, South China University of Technology, Guangzhou 510640, China \\ Correspondence should be addressed to Yuanfei Li; liqfd@163.com
}

Received 8 May 2020; Accepted 4 June 2020; Published 18 July 2020

Academic Editor: Genni Fragnelli

Copyright (๑) 2020 Yuanfei Li. This is an open access article distributed under the Creative Commons Attribution License, which permits unrestricted use, distribution, and reproduction in any medium, provided the original work is properly cited.

In this paper, the initial boundary value problem for the two-dimensional large-scale primitive equations of large-scale oceanic motion in geophysics is considered, which are fundamental models for weather prediction. By establishing rigorous a priori bounds with coefficients and deriving some useful inequalities, the convergence result for the boundary conditions is obtained.

\section{Introduction}

The primitive equations are fundamental models for weather prediction, which are derived from the Boussinesq system of incompressible flow (see, e.g., [1-5]). Due to their importance, many authors have considered the primitive equations analytically by using many new methods (see Zeng [6], Lions and Temam [7, 8], Sun and Cui [9], Hieber et al. [10], and You and Li [11]). For more papers, one can see $[9,12-14]$ and the references therein. It is very obvious that the papers in the literature mainly concern the well posedness of the $2 \mathrm{D}$ or $3 \mathrm{D}$ primitive equations and the properties of solutions.

Different from the results above, the aim of this paper is to establish the convergence result of the solution when the boundary data tend to zero. It is very important to know whether a small change in the equation can cause a large change in the solution. By taking advantage of the mathematical analysis to study these equations, it is helpful to know their applicability in physics. Since some inevitable errors will appear in reality, the study of continuous dependence or convergence results becomes more and more significant. There have been many papers in the literature to study the continuous dependence or convergence for varieties of equations (e.g., Brinkman, Darcy, and Forchheimer equations) (see [15-21]). For some type of primitive equations, one can see $[22,23]$.
In this paper, the two-dimensional large-scale primitive equations (see [24]) are considered

$$
\begin{aligned}
\frac{\partial u}{\partial t}+u \frac{\partial u}{\partial x_{1}}+w \frac{\partial u}{\partial x_{2}}-\frac{1}{\varepsilon^{\prime}} v+\frac{1}{\mathcal{\varepsilon}^{\prime}} \frac{\partial p}{\partial x_{1}} & =\gamma_{1} \Delta u \\
\frac{\partial v}{\partial t}+u \frac{\partial v}{\partial x_{1}}+w \frac{\partial v}{\partial x_{2}}+\frac{1}{\mathcal{\varepsilon}^{\prime}} u & =\gamma_{2} \Delta v, \\
\frac{\partial p}{\partial x_{2}}+\rho & =0, \\
\frac{\partial u}{\partial x_{1}}+\frac{\partial w}{\partial x_{2}} & =0 \\
\frac{\partial T}{\partial t}+u \frac{\partial T}{\partial x_{1}}+w \frac{\partial T}{\partial x_{2}} & =\gamma_{3} \Delta T, \\
\rho & =\rho_{\mathrm{ref}}\left(1-\beta_{T}\left(T-T_{\mathrm{ref}}\right)\right),
\end{aligned}
$$

in a cylindrical domain $\Omega=(0,1) \times(-h, 0), h>0$. In (1), the unknown functions $(u, v), w, \rho, p$, and $T$ are the horizontal velocity field, the vertical velocity, the density, the pressure, and the temperature, respectively; $\varepsilon$ ' is the Rossby number; $\gamma_{i}>0(i=1,2,3)$ are the viscosity coefficients; $\rho_{\text {ref }}$ and $T_{\text {ref }}$ are the reference values of the density and the temperature; $\beta_{T}$ is the expansion coefficient (constants); $\Delta=\partial_{x_{1}}^{2}+\partial_{x_{2}}^{2}$. 
The boundary of $\Omega$ is defined by $\partial \Omega$ which can be partitioned into

$$
\begin{aligned}
\Gamma_{0} & =\left\{\left(x_{1}, x_{2}\right) \in \bar{\Omega}: 0<x_{1}<1, x_{2}=0\right\}, \\
\Gamma_{-h} & =\left\{\left(x_{1}, x_{2}\right) \in \bar{\Omega}: 0<x_{1}<1, x_{2}=-h\right\}, \\
\Gamma_{s} & =\left\{\left(x_{1}, x_{2}\right) \in \bar{\Omega}: x_{1}=0 \text { or } x_{1}=1,-h \leq x_{2} \leq 0\right\} .
\end{aligned}
$$

System (1) also has the following boundary conditions:

$$
\begin{gathered}
\frac{\partial u}{\partial x_{2}}=\alpha_{1} g_{1}\left(x_{1}, t\right), \frac{\partial v}{\partial x_{2}}=\alpha_{2} g_{2}\left(x_{1}, t\right), w=0, \frac{\partial T}{\partial x_{2}}=\beta T^{*}\left(x_{1}, t\right), \\
\text { on } \Gamma_{0}, \\
\frac{\partial u}{\partial x_{2}}=\frac{\partial v}{\partial x_{2}}=0, w=0, \frac{\partial T}{\partial x_{2}}=0, \quad \text { on } \Gamma_{-h}, \\
u=v=w=0, \frac{\partial T}{\partial x_{1}}=0, \quad \text { on } \Gamma_{s},
\end{gathered}
$$

where $g_{1}\left(x_{1}, t\right)$ and $g_{2}\left(x_{1}, t\right)$ are the wind stress on the ocean surface, $\alpha_{1}, \alpha_{2}$, and $\beta$ are the positive constants, and $T^{*}\left(x_{1}, t\right)$ is the typical temperature distribution of the top surface of the ocean. $g_{1}\left(x_{1}, t\right), g_{2}\left(x_{1}, t\right)$, and $T^{*}\left(x_{1}, t\right)$ also satisfy the compatibility boundary conditions:

$$
\begin{aligned}
g_{1}(0, t) & =g_{2}(0, t)=T^{*}(0, t)=g_{1}(1, t)=g_{2}(1, t) \\
& =T^{*}(1, t)=0 .
\end{aligned}
$$

In addition, the initial conditions can be written as

$$
\begin{gathered}
u\left(x_{1}, x_{2}, 0\right)=u_{0}\left(x_{1}, x_{2}\right), \\
v\left(x_{1}, x_{2}, 0\right)=v_{0}\left(x_{1}, x_{2}\right), \\
T\left(x_{1}, x_{2}, 0\right)=T_{0}\left(x_{1}, x_{2}\right), \\
\operatorname{in} \Omega .
\end{gathered}
$$

The present paper is organized as follows. In Section 2, some preliminaries of the problem and some well-known inequalities which will be used in the whole paper are given. Inspired by [25-27], rigorous a priori bounds with coefficients are established. Finally, the convergence result on the boundary data of our problem is derived in Section 4.

\section{Preliminaries of the Problem}

Equations (1)-(5) are formulated as in [7-9]. Realizing the boundary conditions (4), equation $(1)_{4}$ is integrated from $-h$ to $x_{2}$ to obtain

$$
\begin{aligned}
& w\left(x_{1}, x_{2}, t\right)=w\left(x_{1},-h, t\right)-\int_{-h}^{x_{2}} \frac{\partial}{\partial x_{1}} u\left(x_{1}, \zeta, t\right) \mathrm{d} \zeta \\
& =-\frac{\partial}{\partial x_{1}} \int_{-h}^{x_{2}} u\left(x_{1}, \zeta, t\right) \mathrm{d} \zeta \\
& \int_{-h}^{0} \frac{\partial}{\partial x_{1}} u\left(x_{1}, \zeta, t\right) \mathrm{d} \zeta=-\frac{\partial}{\partial x_{1}} \int_{-h}^{0} u\left(x_{1}, \zeta, t\right) \mathrm{d} \zeta=0
\end{aligned}
$$

By integrating $(1)_{3}$ and $(1)_{4}$, the following is obtained:

$$
\begin{aligned}
p\left(x_{1}, x_{2}, t\right)= & p_{s}-\int_{x_{2}}^{0} \rho\left(x_{1}, \zeta, t\right) \mathrm{d} \zeta=p_{s} \\
& -\rho_{\text {ref }} \int_{x_{2}}^{0}\left[1-\beta_{T}\left(T\left(x_{1}, \zeta, t\right)-T_{\text {ref }}\right)\right] \mathrm{d} \zeta,
\end{aligned}
$$

where $p_{s}=p\left(x_{1}, 0, t\right)$ is the pressure on the surface of the ocean. For convenience, suppose $\rho_{\text {ref }} \beta_{T}=\varepsilon^{\prime}=$ $\gamma_{1}=\gamma_{2}=\gamma_{3}=1$. Inserting (6) and (7) into (1)-(5), the problem can be rewritten as

$$
\begin{aligned}
& \frac{\partial u}{\partial t}+u \frac{\partial u}{\partial x_{1}}-\left(\int_{-h}^{x_{2}} \frac{\partial}{\partial x_{1}} u\left(x_{1}, \zeta, t\right) \mathrm{d} \zeta\right) \frac{\partial u}{\partial x_{2}}-v \\
& +\frac{\partial p_{s}}{\partial x_{1}}-\left(\int_{x_{2}}^{0} \frac{\partial}{\partial x_{1}} T\left(x_{1}, \zeta, t\right) \mathrm{d} \zeta\right)=\Delta u, \\
& \frac{\partial v}{\partial t}+u \frac{\partial v}{\partial x_{1}}-\left(\int_{-h}^{x_{2}} \frac{\partial}{\partial x_{1}} u\left(x_{1}, \zeta, t\right) \mathrm{d} \zeta\right) \frac{\partial v}{\partial x_{2}}+u=\Delta v, \\
& \frac{\partial T}{\partial t}+u \frac{\partial T}{\partial x_{1}}-\left(\int_{-h}^{x_{2}} \frac{\partial}{\partial x_{1}} u\left(x_{1}, \zeta, t\right) \mathrm{d} \zeta\right) \frac{\partial T}{\partial x_{2}}=\Delta T,
\end{aligned}
$$

with the following boundary conditions:

$$
\begin{aligned}
& \left.\frac{\partial u}{\partial x_{2}}\right|_{x_{2}=0}=\alpha_{1} g_{1}\left(x_{1}, t\right), \\
& \left.\frac{\partial v}{\partial x_{2}}\right|_{x_{2}=0}=\alpha_{2} g_{2}\left(x_{1}, t\right), \\
& \left.\frac{\partial u}{\partial x_{2}}\right|_{x_{2}=-h}=\left.\frac{\partial v}{\partial x_{2}}\right|_{x_{2}=-h}=0, \\
& \left.\frac{\partial T}{\partial x_{2}}\right|_{x_{2}=0}=-\beta T_{\Gamma_{s}}=0 \\
& \left.\frac{\partial T}{\partial x_{2}}\right|_{x_{2}=-h}=0 \\
& \left.\frac{\partial T}{\partial x_{1}}\right|_{\Gamma_{s}}=0
\end{aligned}
$$

and the initial conditions:

$$
\left.(u, v, T)\right|_{t=0}=\left(u_{0}, v_{0}, T_{0}\right) .
$$

In this paper, some well-known inequalities are used throughout this paper.

Lemma 1. If $\omega\left(x_{1}\right) \in C^{1}(0,1)$ and $\omega(0)=\omega(1)=0$, then

$$
\int_{0}^{1} \omega^{2} \mathrm{~d} x_{1} \leq \frac{1}{\pi^{2}} \int_{0}^{1}\left(\frac{\partial \omega}{\partial x_{1}}\right)^{2} \mathrm{~d} x_{1} .
$$


Lemma 2. If $\omega\left(x_{2}\right) \in C^{1}(-h, 0)$ and $\omega(-h)=\omega(0)=0$, then

$$
\int_{-h}^{0} \omega^{2} \mathrm{~d} x_{2} \leq \frac{h^{2}}{\pi^{2}} \int_{-h}^{0}\left(\frac{\partial \omega}{\partial x_{2}}\right)^{2} \mathrm{~d} x_{2}
$$

For proofs of these inequalities, see references [28, 29].

Lemma 3. If $\omega\left(x_{1}, x_{2}, t\right)$ is a sufficiently smooth function in $\Omega=(0,1) \times(-h, 0)$ and $\omega\left(0, x_{2}, t\right)=\omega\left(1, x_{2}, t\right)=0$, then

$$
\begin{aligned}
\left(\int_{\Omega} \omega^{4} \mathrm{~d} A\right)^{1 / 2} \leq & C\left[\left(\int_{\Omega} \omega^{2} \mathrm{~d} A\right)^{1 / 2}\left(\int_{\Omega}|\nabla \omega|^{2} \mathrm{~d} A\right)^{1 / 2}\right. \\
& \left.+\left(\int_{\Omega} \omega^{2} \mathrm{~d} A\right)^{1 / 4}\left(\int_{\Omega}|\nabla \omega|^{2} \mathrm{~d} A\right)^{3 / 4}\right],
\end{aligned}
$$

or

$$
\left(\int_{\Omega} \omega^{4} \mathrm{~d} A\right)^{1 / 2} \leq C\left[\int_{\Omega} \omega^{2} \mathrm{~d} A+\delta \int_{\Omega}|\nabla \omega|^{2} \mathrm{~d} A\right],
$$

where $\nabla=\left(\partial_{x_{1}}, \partial_{x_{2}}\right), C$ is a positive computable constant, and $\delta$ is a positive arbitrary constant.

Proof. By the Hölder inequality, one can write

$$
\int_{\Omega} \omega^{4} \mathrm{~d} A \leq \int_{-h}^{0}\left(\int_{0}^{1} \omega^{6} \mathrm{~d} x_{1}\right)^{1 / 2}\left(\int_{0}^{1} \omega^{2} \mathrm{~d} x_{1}\right)^{1 / 2} \mathrm{~d} x_{2} .
$$

Since $\omega\left(0, x_{2}, t\right)=\omega\left(1, x_{2}, t\right)=0$, the following is obtained:

$$
\begin{aligned}
\omega^{3} & =3 \int_{0}^{x_{1}} \omega^{2}\left(\xi, x_{2}, t\right) \frac{\partial \omega\left(\xi, x_{2}, t\right)}{\partial \xi} \mathrm{d} \xi \\
& =-3 \int_{x_{1}}^{1} \omega^{2}\left(\xi, x_{2}, t\right) \frac{\partial \omega\left(\xi, x_{2}, t\right)}{\partial \xi} \mathrm{d} \xi .
\end{aligned}
$$

Therefore,

$$
|\omega|^{3} \leq \frac{3}{2} \int_{0}^{1} \omega^{2}\left(x_{1}, x_{2}, t\right)\left|\frac{\partial \omega\left(x_{1}, x_{2}, t\right)}{\partial x_{1}}\right| \mathrm{d} x_{1} .
$$

Then,

$$
\left(\int_{0}^{1} \omega^{6} \mathrm{~d} x_{1}\right)^{1 / 2} \leq \frac{3}{2}\left(\int_{0}^{1} \omega^{2}\left|\frac{\partial \omega}{\partial x_{1}}\right| \mathrm{d} x_{1}\right) .
$$

Inserting (19) into (16), the following is obtained:

$$
\begin{aligned}
\int_{\Omega} \omega^{4} \mathrm{~d} A & \leq \frac{3}{2} \int_{-h}^{0}\left(\int_{0}^{1} \omega^{2}\left|\frac{\partial \omega}{\partial x_{1}}\right| \mathrm{d} x_{1}\right)\left(\int_{0}^{1} \omega^{2} \mathrm{~d} x_{1}\right)^{1 / 2} \mathrm{~d} x_{2} \\
& \leq \frac{3}{2} \max _{-h \leq x_{2} \leq 0}\left\{\left(\int_{0}^{1} \omega^{2} \mathrm{~d} x_{1}\right)^{1 / 2}\right\} \int_{\Omega} \omega^{2}\left|\frac{\partial \omega}{\partial x_{1}}\right| \mathrm{d} A .
\end{aligned}
$$

Obviously,

$$
\begin{aligned}
\omega^{2} & =2 \int_{-h}^{x_{2}} \omega\left(x_{1}, \zeta, t\right) \frac{\partial \omega\left(x_{1}, \zeta, t\right)}{\partial \zeta} \mathrm{d} \zeta+\omega^{2}\left(x_{1},-h, t\right) \\
& =-2 \int_{x_{2}}^{0} \omega\left(x_{1}, \zeta, t\right) \frac{\partial \omega\left(x_{1}, \zeta, t\right)}{\partial \zeta} \mathrm{d} \zeta+\omega^{2}\left(x_{1}, 0, t\right),
\end{aligned}
$$

so,

$$
\omega^{2} \leq \int_{-h}^{0}|\omega|\left|\frac{\partial \omega}{\partial x_{2}}\right| \mathrm{d} x_{2}+\frac{1}{2}\left[\omega^{2}\left(x_{1}, 0, t\right)+\omega^{2}\left(x_{1},-h, t\right)\right] .
$$

To bound the last term of (22), a new known function $f\left(x_{2}\right)$ is defined, satisfying

$$
\begin{aligned}
f(0) & >0, \\
f(-h) & <0, \\
\left|f^{\prime}\left(x_{2}\right)\right| & \leq m_{1}, \\
\left|f\left(x_{2}\right)\right| & \leq m_{2}, \\
\text { for }-h & \leq x_{2} \leq 0,
\end{aligned}
$$

where $m_{1}$ and $m_{2}$ are the positive constants. For example, $f\left(x_{2}\right)=\left(m_{1} / 2\right)\left(\left(x_{2}+(h / 2)\right), m_{1} h<4 m_{2}\right.$, satisfies all the conditions in (23). Using the above estimates and employing the divergence theorem allow one to write

$$
\begin{aligned}
& \min \{f(0),-f(-h)\}\left(\left[\omega^{2}\left(x_{1}, 0, t\right)+\omega^{2}\left(x_{1},-h, t\right)\right]\right. \\
& \leq f(0) \omega^{2}\left(x_{1}, 0, t\right)-f(-h) \omega^{2}\left(x_{1},-h, t\right) \\
& =\int_{-h}^{0} \frac{\partial}{\partial x_{2}}\left(f \omega^{2}\right) \mathrm{d} x_{2}=\int_{-h}^{0} f^{\prime}\left(x_{2}\right) \omega^{2} \mathrm{~d} x_{2}+2 \int_{-h}^{0} f \omega \frac{\partial \omega}{\partial x_{2}} \mathrm{~d} x_{2} \\
& \leq m_{1} \int_{-h}^{0} \omega^{2} \mathrm{~d} x_{2}+2 m_{2} \int_{-h}^{0}|\omega|\left|\frac{\partial \omega}{\partial x_{2}}\right| \mathrm{d} x_{2} .
\end{aligned}
$$

Inserting (24) into (22),

$$
\omega^{2} \leq m_{3} \int_{-h}^{0} \omega^{2} \mathrm{~d} x_{2}+m_{4} \int_{-h}^{0}|\omega|\left|\frac{\partial \omega}{\partial x_{2}}\right| \mathrm{d} x_{2},
$$

where

$$
\begin{aligned}
& m_{3}=\frac{m_{1}}{2 \min \{f(0),-f(-h)\}}, \\
& m_{4}=1+\frac{m_{2}}{\min \{f(0),-f(-h)\}} .
\end{aligned}
$$

Therefore,

$$
\max _{-h \leq x_{2} \leq 0}\left\{\left(\int_{0}^{1} \omega^{2} \mathrm{~d} x_{1}\right)^{1 / 2}\right\} \leq\left(m_{3} \int_{\Omega} \omega^{2} \mathrm{~d} A+m_{4} \int_{\Omega}|\omega|\left|\frac{\partial \omega}{\partial x_{2}}\right| \mathrm{d} A\right)^{1 / 2} .
$$

Thus, from (20) and (27) and by the Hölder inequality, one has 


$$
\begin{aligned}
\int_{\Omega} \omega^{4} \mathrm{~d} A \leq & \frac{3}{2}\left[m_{3} \int_{\Omega} \omega^{2} \mathrm{~d} A+m_{4}\left(\int_{\Omega} \omega^{2} \mathrm{~d} A\right)^{1 / 2}\right. \\
& \left.\cdot\left(\int_{\Omega}\left|\frac{\partial \omega}{\partial x_{2}}\right|^{2} \mathrm{~d} A\right)^{1 / 2}\right]^{1 / 2}\left(\int_{\Omega} \omega^{4} \mathrm{~d} A\right)^{1 / 2} \\
& \cdot\left(\int_{\Omega}\left|\frac{\partial \omega}{\partial x_{1}}\right|^{2} \mathrm{~d} A\right)^{1 / 2} .
\end{aligned}
$$

After simplification,

$$
\begin{aligned}
\left(\int_{\Omega} \omega^{4} \mathrm{~d} A\right)^{1 / 2} \leq & C\left[\left(\int_{\Omega} \omega^{2} \mathrm{~d} A\right)^{1 / 2}\left(\int_{\Omega}|\nabla \omega|^{2} \mathrm{~d} A\right)^{1 / 2}\right. \\
& \left.+\left(\int_{\Omega} \omega^{2} \mathrm{~d} A\right)^{1 / 4}\left(\int_{\Omega}|\nabla \omega|^{2} \mathrm{~d} A\right)^{3 / 4}\right] .
\end{aligned}
$$

\section{A Priori Estimates}

Now, some a priori estimates for the solutions of (9)-(11) are derived.

Lemma 4. Assume $T$ be the solutions to (9)-(11) with $T_{0} \in L_{2}(\Omega), T^{*} \in H^{1}(\Omega)$. Then,

$$
\int_{0}^{t} \int_{\Omega}|\nabla T|^{2} \mathrm{~d} A \mathrm{~d} \eta \leq F_{1}(t)
$$

where $F_{1}(t)$ will be defined later.

Proof. Taking the inner product of equation $(9)_{3}$ with $T$, in $L^{2}(\Omega)$, the following is obtained:

$$
\begin{gathered}
\frac{1}{2} \frac{\mathrm{d}}{\mathrm{d} t} \int_{\Omega} T^{2} \mathrm{~d} A+\int_{\Omega}|\nabla T|^{2} \mathrm{~d} A=\left.\int_{0}^{1} \frac{\partial T}{\partial x_{2}}\right|_{x_{2}=0} T\left(x_{1}, 0, t\right) \mathrm{d} x_{1} \\
-\int_{\Omega}\left[u \frac{\partial T}{\partial x_{1}}-\left(\int_{-h}^{x_{2}} \frac{\partial}{\partial x_{1}} u\left(x_{1}, \zeta, t\right) \mathrm{d} \zeta\right) \frac{\partial T}{\partial x_{2}}\right] T \mathrm{~d} A
\end{gathered}
$$

A function $H\left(x_{1}, x_{2}, t\right)$ is defined as

$$
\frac{\partial H}{\partial x_{2}}=\frac{\beta\left(x_{2}+h\right)}{h} T^{*}\left(x_{1}, t\right) \text {. }
$$

Therefore,

$$
\begin{aligned}
\left.\int_{0}^{1} \frac{\partial T}{\partial x_{2}}\right|_{x_{2}=0} T\left(x_{1}, 0, t\right) \mathrm{d} x_{1}= & \left.\int_{0}^{1} \frac{\partial H}{\partial x_{2}}\right|_{x_{2}=0} T\left(x_{1}, 0, t\right) \mathrm{d} x_{1} \\
= & \int_{\Omega} \frac{\partial}{\partial x_{2}}\left(\frac{\partial H}{\partial x_{2}} T\right) \mathrm{d} A \\
= & \int_{\Omega} \frac{\partial^{2} H}{\partial x_{2}^{2}} T \mathrm{~d} A+\int_{\Omega} \frac{\partial H}{\partial x_{2}} \frac{\partial T}{\partial x_{2}} \mathrm{~d} A \\
= & \int_{\Omega} \frac{\partial^{2} H}{\partial x_{2}^{2}} T \mathrm{~d} A+\int_{\Omega} \frac{\partial H}{\partial x_{2}} \frac{\partial T}{\partial x_{2}} \mathrm{~d} A \\
& +\frac{1}{2} \int_{\Omega}\left(\frac{\partial H}{\partial x_{2}}\right)^{2} \mathrm{~d} A+\frac{1}{2} \int_{\Omega}\left(\frac{\partial T}{\partial x_{2}}\right)^{2} \mathrm{~d} A .
\end{aligned}
$$

Integrating by parts,

$$
-\int_{\Omega}\left[u \frac{\partial T}{\partial x_{1}}-\left(\int_{-h}^{x_{2}} \frac{\partial}{\partial x_{1}} u\left(x_{1}, \zeta, t\right) \mathrm{d} \zeta\right) \frac{\partial T}{\partial x_{2}}\right] T \mathrm{~d} A=0 .
$$

By the above results, the following is obtained:

$$
\frac{\mathrm{d}}{\mathrm{d} t} \int_{\Omega} T^{2} \mathrm{~d} A+\int_{\Omega}|\nabla T|^{2} \mathrm{~d} A=\int_{\Omega} T^{2} \mathrm{~d} A+a_{1}(t)
$$

where $a_{1}(t)=\int_{\Omega}\left(\partial^{2} H / \partial x_{2}^{2}\right)^{2} \mathrm{~d} A+\int_{\Omega}\left(\partial H / \partial x_{2}\right)^{2} \mathrm{~d} A$. Using inequality (35) and the Gronwall inequality, the following is obtained:

$$
\int_{\Omega} T^{2} \mathrm{~d} A \leq \int_{\Omega} T_{0}^{2} \mathrm{~d} A \cdot e^{t}+\int_{0}^{t} a_{1}(\eta) e^{t-\eta} \mathrm{d} \eta \doteq a_{2}(t) .
$$

Moreover,

$$
\int_{0}^{t} \int_{\Omega}|\nabla T|^{2} \mathrm{~d} A \mathrm{~d} \eta \leq F_{1}(t)
$$

where $F_{1}(t)=\int_{0}^{t}\left[a_{1}(\eta)+a_{2}(\eta)\right] \mathrm{d} \eta+\int_{\Omega} T_{0}^{2} \mathrm{~d} A$.

Lemma 5. Let $(u, v)$ be the solution of (9)-(11) with $u_{0}, v_{0}, T_{0} \in L_{2}(\Omega)$. Then,

$$
\begin{array}{r}
\int_{\Omega} u^{2} \mathrm{~d} A+\int_{\Omega} v^{2} \mathrm{~d} A \leq F_{2}(t), \\
\int_{0}^{t} \int_{\Omega}|\nabla u|^{2} \mathrm{~d} A \mathrm{~d} \eta+\int_{0}^{t} \int_{\Omega}|\nabla v|^{2} \mathrm{~d} A \mathrm{~d} \eta \leq F_{3}(t),
\end{array}
$$

where $F_{2}(t)$ and $F_{3}(t)$ will be defined later.

Proof. Taking the inner product of equation (9) ${ }_{1}$ with $u$, in $L^{2}(\Omega)$, the following is obtained:

$$
\begin{aligned}
\frac{1}{2} \frac{\mathrm{d}}{\mathrm{d} t} \int_{\Omega} u^{2} \mathrm{~d} A+\int_{\Omega}|\nabla u|^{2} \mathrm{~d} A= & \left.\int_{0}^{1} \frac{\partial u}{\partial x_{2}}\right|_{x_{2}=0} u\left(x_{1}, 0, t\right) \mathrm{d} x_{1} \\
& -\int_{\Omega} u v \mathrm{~d} A \\
& +\int_{\Omega}\left(\int_{x_{2}}^{0} \frac{\partial}{\partial x_{1}} T\left(x_{1}, \zeta, t\right) \mathrm{d} \zeta\right) u \mathrm{~d} A .
\end{aligned}
$$

A function $S_{1}\left(x_{1}, x_{2}, t\right)$ is defined as

$$
\frac{\partial S_{1}}{\partial x_{2}}=\frac{\alpha_{1}\left(x_{2}+h\right)}{h} g_{1}\left(x_{1}, t\right) \text {. }
$$

Obviously, $S_{1}$ has the same boundary conditions of $u$. Therefore, 


$$
\begin{aligned}
\left.\int_{0}^{1} \frac{\partial u}{\partial x_{2}}\right|_{x_{2}=0} u\left(x_{1}, 0, t\right) \mathrm{d} x_{1}= & \left.\int_{0}^{1} \frac{\partial S_{1}}{\partial x_{2}}\right|_{x_{2}=0} u\left(x_{1}, 0, t\right) \mathrm{d} x_{1}=\int_{\Omega} \frac{\partial}{\partial x_{2}}\left(\frac{\partial S_{1}}{\partial x_{2}} u\right) \mathrm{d} A \\
& =\int_{\Omega} \frac{\partial^{2} S_{1}}{\partial x_{2}^{2}} u \mathrm{~d} A+\int_{\Omega} \frac{\partial S_{1}}{\partial x_{2}} \frac{\partial u}{\partial x_{2}} \mathrm{~d} A \\
\leq & \frac{1}{2} \int_{\Omega}\left(\frac{\partial^{2} S_{1}}{\partial x_{2}^{2}}\right)^{2} \mathrm{~d} A+\frac{1}{2} \int_{\Omega} u^{2} \mathrm{~d} A \\
& +\frac{1}{2} \int_{\Omega}\left(\frac{\partial S_{1}}{\partial x_{2}}\right)^{2} \mathrm{~d} A+\frac{1}{2} \int_{\Omega}\left(\frac{\partial u}{\partial x_{2}}\right)^{2} \mathrm{~d} A
\end{aligned}
$$

By the Cauchy-Schwarz inequality, the following is obtained:

$$
\begin{aligned}
\int_{\Omega}\left(\int_{x_{2}}^{0} \frac{\partial}{\partial x_{1}} T\left(x_{1}, \zeta, t\right) \mathrm{d} \zeta\right) u \mathrm{~d} A \leq & {\left[\int_{\Omega}\left(\frac{\partial T}{\partial x_{1}}\right)^{2} \mathrm{~d} A\right]^{1 / 2} } \\
& \cdot\left[\int_{\Omega} u^{2} \mathrm{~d} A\right]^{1 / 2} \\
\leq & \frac{h}{2} \int_{\Omega}\left(\frac{\partial T}{\partial x_{1}}\right)^{2} \mathrm{~d} A \\
& +\frac{h}{2} \int_{\Omega} u^{2} \mathrm{~d} A
\end{aligned}
$$

By the above results, the following is obtained:

$$
\begin{aligned}
& \frac{\mathrm{d}}{\mathrm{d} t} \int_{\Omega} u^{2} \mathrm{~d} A+\int_{\Omega}|\nabla u|^{2} \mathrm{~d} A \leq-2 \int_{\Omega} u v \mathrm{~d} A+(1+h) \int_{\Omega} u^{2} \mathrm{~d} A \\
& \quad+h \int_{\Omega}\left(\frac{\partial T}{\partial x_{1}}\right)^{2} \mathrm{~d} A+\int_{\Omega}\left(\frac{\partial^{2} S_{1}}{\partial x_{2}^{2}}\right)^{2} \mathrm{~d} A+\int_{\Omega}\left(\frac{\partial S_{1}}{\partial x_{2}}\right)^{2} \mathrm{~d} A .
\end{aligned}
$$

Similarly, from $(9)_{2}$,

$$
\begin{gathered}
\frac{\mathrm{d}}{\mathrm{d} t} \int_{\Omega} v^{2} \mathrm{~d} A+\int_{\Omega}|\nabla v|^{2} \mathrm{~d} A \leq 2 \int_{\Omega} u v \mathrm{~d} A+\int_{\Omega} v^{2} \mathrm{~d} A \\
\quad+\int_{\Omega}\left(\frac{\partial^{2} S_{2}}{\partial x_{2}^{2}}\right)^{2} \mathrm{~d} A+\int_{\Omega}\left(\frac{\partial S_{2}}{\partial x_{2}}\right)^{2} \mathrm{~d} A,
\end{gathered}
$$

where

$$
\frac{\partial S_{2}}{\partial x_{2}}=\frac{\alpha_{1}\left(x_{2}+h\right)}{h} g_{2}\left(x_{1}, t\right)
$$

Combining (43) and (44), the following is obtained: $\frac{\mathrm{d}}{\mathrm{d} t}\left(\int_{\Omega} u^{2} \mathrm{~d} A+\int_{\Omega} v^{2} \mathrm{~d} A\right)+\int_{\Omega}|\nabla u|^{2} \mathrm{~d} A+\int_{\Omega}|\nabla v|^{2} \mathrm{~d} A$

$$
\leq(1+h)\left(\int_{\Omega} u^{2} \mathrm{~d} A+\int_{\Omega} v^{2} \mathrm{~d} A\right)+h \int_{\Omega}\left(\frac{\partial T}{\partial x_{1}}\right)^{2} \mathrm{~d} A+a_{3}(t)
$$

where $\quad a_{3}(t)=\int_{\Omega}\left(\partial^{2} S_{1} / \partial x_{2}^{2}\right)^{2} \mathrm{~d} A+\int_{\Omega}\left(\partial S_{1} / \partial x_{2}\right)^{2} \mathrm{~d} A+$ $\int_{\Omega}\left(\partial^{2} S_{2} / \partial x_{2}^{2}\right)^{2} \mathrm{~d} A+\int_{\Omega}\left(\partial S_{2} / \partial x_{2}\right)^{2} \mathrm{~d} A$. By the Gronwall inequality and Lemma 2 , one gets

$$
\int_{\Omega} u^{2} \mathrm{~d} A+\int_{\Omega} v^{2} \mathrm{~d} A \leq F_{2}(t)
$$

where $F_{2}(t)=h e^{(1+h) t}\left(\int_{\Omega} u_{0}^{2} \mathrm{~d} A+\int_{\Omega} v_{0}^{2} \mathrm{~d} A+F_{1}(t)\right)+\int_{0}^{t} a_{3}$ $(\eta) e^{(1+h)(t-\eta)} \mathrm{d} \eta$. Moreover,

$$
\int_{0}^{t} \int_{\Omega}|\nabla u|^{2} \mathrm{~d} A \mathrm{~d} \eta+\int_{0}^{t} \int_{\Omega}|\nabla v|^{2} \mathrm{~d} A \mathrm{~d} \eta \leq F_{3}(t)
$$

where $F_{3}(t)=(1+h) \int_{0}^{t} F_{2}(\eta) \mathrm{d} \eta+h F_{1}(t)+\int_{0}^{t} a_{3}(\eta) \mathrm{d} \eta+$ $\int_{\Omega} u_{0}^{2} \mathrm{~d} A+\int_{\Omega} v_{0}^{2} \mathrm{~d} A$.

Lemma 6. If $T_{0}, T^{*} \in L_{\infty}(\Omega)$, then the solution $T$ of $(2.4)_{3}$ satisfies

$$
\sup _{\Omega}|T| \leq T_{m}
$$

where $T_{m}=\sup _{\Omega}\left\{\left\|T^{*}\right\|_{\infty},\left\|T_{0}\right\|_{\infty}\right\}$.

Proof. Multiplying $(2.4)_{3}$ by $T^{p-1}$ and integrating by parts, one gets

$$
\begin{gathered}
\frac{1}{p} \frac{\mathrm{d}}{\mathrm{d} t} \int_{\Omega} T^{p} \mathrm{~d} A+\frac{p-1}{p^{2}} \int_{\Omega}\left|\nabla T^{p / 2}\right|^{2} \mathrm{~d} A \\
\quad=\left.\int_{0}^{1} \frac{\partial T}{\partial x_{2}}\right|_{x_{2}=0} T^{p-1}\left(x_{1}, 0, t\right) \mathrm{d} x_{1} .
\end{gathered}
$$

By the Hölder inequality and the Cauchy-Schwarz inequality, the following is obtained: 


$$
\begin{aligned}
& \left.\int_{0}^{1} \frac{\partial T}{\partial x_{2}}\right|_{x_{2}=0} T^{p-1}\left(x_{1}, 0, t\right) \mathrm{d} x=\int_{0}^{1} \frac{\partial H}{\partial x_{2}} T^{p-1} \mathrm{~d} x_{1} \\
& =\int_{\Omega} \frac{\partial}{\partial x_{2}}\left(\frac{\partial H}{\partial x_{2}} T^{p-1}\right) d A \\
& =\int_{\Omega} \frac{\partial^{2} H}{\partial x_{2}^{2}} T^{p-1} \mathrm{~d} A \\
& +(p-1) \int_{\Omega} \frac{\partial H}{\partial x_{2}} T^{p-2} \frac{\partial T}{\partial x_{2}} \mathrm{~d} A \\
& \leq \frac{1}{p} \int_{\Omega}\left(\frac{\partial^{2} H}{\partial x_{2}^{2}}\right)^{p} \mathrm{~d} A \\
& +\frac{(p-1)}{p} \int_{\Omega} T^{p} \mathrm{~d} A \\
& +\frac{2(p-1)}{p} \int_{\Omega}\left(\frac{\partial H}{\partial x_{2}}\right)^{p} \mathrm{~d} A \\
& +\frac{(p-1)(p-2)}{p} \int_{\Omega} T^{p} \mathrm{~d} A \\
& +\frac{(p-1)}{p^{2}} \int_{\Omega}\left|\nabla T^{p / 2}\right|^{2} \mathrm{~d} A .
\end{aligned}
$$

Therefore, from (50) and (51), one has

$$
\begin{aligned}
\frac{\mathrm{d}}{\mathrm{d} t} \int_{\Omega} T^{p} \mathrm{~d} A \leq & (p-1)^{2} \int_{\Omega} T^{p} \mathrm{~d} A+2(p-1) \int_{\Omega}\left(\frac{\partial H}{\partial x_{2}}\right)^{p} \mathrm{~d} A \\
& +(p-1)(p-2) \int_{\Omega}\left(\frac{\partial^{2} H}{\partial x_{2}^{2}}\right)^{p} \mathrm{~d} A
\end{aligned}
$$

By the Gronwall inequality, the following is obtained:

$$
\begin{aligned}
& \int_{\Omega} T^{p} \mathrm{~d} A \leq \int_{\Omega} T_{0}^{p} \mathrm{~d} A \cdot e^{(p-1)^{2} t}+\int_{0}^{t} e^{(p-1)^{2}(t-\eta)} \\
& \cdot\left[2(p-1) \int_{\Omega}\left(\frac{\partial H}{\partial x_{2}}\right)^{p} \mathrm{~d} A+(p-1)(p-2) \int_{\Omega}\left(\frac{\partial^{2} H}{\partial x_{2}^{2}}\right)^{p} \mathrm{~d} A\right] \mathrm{d} \eta .
\end{aligned}
$$

Therefore,

$$
\begin{aligned}
& \left(\int_{\Omega} T^{p} \mathrm{~d} A\right)^{1 / p} \leq\left\{\int_{\Omega} T_{0}^{p} d A \cdot e^{(p-1)^{2} t}+\int_{0}^{t} e^{(p-1)^{2}(t-\eta)}\right. \\
& \left.\quad \cdot\left[2(p-1) \int_{\Omega}\left(\frac{\partial H}{\partial x_{2}}\right)^{p} \mathrm{~d} A+(p-1)(p-2) \int_{\Omega}\left(\frac{\partial^{2} H}{\partial x_{2}^{2}}\right)^{p} \mathrm{~d} A\right] \mathrm{d} \eta\right\}^{1 / p} .
\end{aligned}
$$

Letting now $p \longrightarrow \infty$ in (54), one obtains

$$
\sup _{\Omega}|T| \leq T_{m}
$$

Lemma 7. Let $(u, v)$ be the solution of (9)-(11) with $u_{0}, v_{0}, T_{0} \in H^{1}(\Omega)$. Then,

$$
\begin{aligned}
& \int_{\Omega}\left(\frac{\partial u}{\partial x_{2}}\right)^{2} \mathrm{~d} A+\int_{\Omega}\left(\frac{\partial v}{\partial x_{2}}\right)^{2} \mathrm{~d} A+\int_{0}^{t} \int_{\Omega}\left|\nabla \frac{\partial u}{\partial x_{2}}\right|^{2} \mathrm{~d} A \mathrm{~d} \eta \\
& +\int_{0}^{t} \int_{\Omega}\left|\nabla \frac{\partial v}{\partial x_{2}}\right|^{2} \mathrm{~d} A \mathrm{~d} \eta \leq F_{5}(t) .
\end{aligned}
$$

Moreover,

$$
\int_{0}^{t} \int_{\Omega}\left(\frac{\partial u}{\partial x_{2}}\right)^{4} \mathrm{~d} A \mathrm{~d} \eta+\int_{0}^{t} \int_{\Omega}\left(\frac{\partial v}{\partial x_{2}}\right)^{4} \mathrm{~d} A \mathrm{~d} \eta \leq F_{6}(t)
$$

where $F_{5}(t)$ and $F_{6}(t)$ will be defined later.

Proof. Using $(2.4)_{1}$, one starts with

$$
\begin{gathered}
\int_{0}^{t} \int_{\Omega} \frac{\partial}{\partial x_{2}}\left\{\frac{\partial u}{\partial t}+u \frac{\partial u}{\partial x_{1}}-\left(\int_{-h}^{x_{2}} \frac{\partial}{\partial x_{1}} u\left(x_{1}, \zeta, t\right) \mathrm{d} \zeta\right) \frac{\partial u}{\partial x_{2}}\right. \\
\left.-v+\frac{\partial p_{s}}{\partial x_{1}}-\left(\int_{x_{2}}^{0} \frac{\partial}{\partial x_{1}} T\left(x_{1}, \zeta, t\right) \mathrm{d} \zeta\right)-\Delta u\right\} \frac{\partial u}{\partial x_{2}} \mathrm{~d} A \mathrm{~d} \eta=0 .
\end{gathered}
$$

Integrating by parts, the following is obtained: 
Discrete Dynamics in Nature and Society

7

$$
\begin{aligned}
& \frac{1}{2} \int_{\Omega}\left(\frac{\partial u}{\partial x_{2}}\right)^{2} \mathrm{~d} A+\int_{0}^{t} \int_{\Omega}\left|\nabla \frac{\partial u}{\partial x_{2}}\right|^{2} \mathrm{~d} A \mathrm{~d} \eta \\
& =\int_{0}^{t} \int_{0}^{1} \frac{\partial u}{\partial x_{2}} \frac{\partial^{2} u}{\partial x_{2}^{2}} \mathrm{~d} x_{1} \mathrm{~d} \eta+\frac{1}{2} \int_{\Omega}\left(\frac{\partial u_{0}}{\partial x_{2}}\right)^{2} \mathrm{~d} A \\
& \quad+\int_{0}^{t} \int_{\Omega} \frac{\partial v}{\partial x_{2}} \frac{\partial u}{\partial x_{2}} \mathrm{~d} A \mathrm{~d} \eta-\int_{0}^{t} \int_{\Omega} \frac{\partial T}{\partial x_{1}} \frac{\partial u}{\partial x_{2}} \mathrm{~d} A \mathrm{~d} \eta
\end{aligned}
$$

By Lemmas 4 and 5 and the Hölder inequality, one has

$$
\begin{aligned}
-\int_{0}^{t} \int_{\Omega} \frac{\partial T}{\partial x_{1}} \frac{\partial u}{\partial x_{2}} \mathrm{~d} A \mathrm{~d} \eta & \leq\left(\int_{0}^{t} \int_{\Omega}\left(\frac{\partial T}{\partial x_{1}}\right)^{2} \mathrm{~d} A \mathrm{~d} \eta\right)^{1 / 2} \\
& \cdot\left(\int_{0}^{t} \int_{\Omega}\left(\frac{\partial u}{\partial x_{2}}\right)^{2} \mathrm{~d} A \mathrm{~d} \eta\right)^{1 / 2} \\
& \leq \sqrt{F_{1}(t) F_{3}(t)}
\end{aligned}
$$

By (40) and using the divergence theorem,

$$
\begin{aligned}
& \int_{0}^{t} \int_{0}^{1} \frac{\partial u}{\partial x_{2}} \frac{\partial^{2} u}{\partial x_{2}^{2}} \mathrm{~d} x_{1} \mathrm{~d} \eta=\int_{0}^{t} \int_{\Omega} \frac{\partial}{\partial x_{2}}\left(\frac{\partial S_{1}}{\partial x_{2}} \frac{\partial^{2} u}{\partial x_{2}^{2}}\right) \mathrm{d} A \mathrm{~d} \eta \\
& =\int_{0}^{t} \int_{\Omega} \frac{\partial^{2} S_{1}}{\partial x_{2}^{2}} \frac{\partial^{2} u}{\partial x_{2}^{2}} \mathrm{~d} A \mathrm{~d} \eta+\int_{0}^{t} \int_{\Omega} \frac{\partial S_{1}}{\partial x_{2}} \frac{\partial^{3} u}{\partial x_{2}^{3}} \mathrm{~d} A \mathrm{~d} \eta \\
& \leq \frac{1}{2} \int_{0}^{t} \int_{\Omega}\left(\frac{\partial^{2} S_{1}}{\partial x_{2}^{2}}\right)^{2} \mathrm{~d} A \mathrm{~d} \eta+\frac{1}{2} \int_{0}^{t} \int_{\Omega}\left(\frac{\partial^{2} u}{\partial x_{2}^{2}}\right)^{2} \mathrm{~d} A \mathrm{~d} \eta \\
& +\int_{0}^{t} \int_{\Omega} \frac{\partial S_{1}}{\partial x_{2}} \frac{\partial}{\partial x_{2}}\left\{\frac{\partial u}{\partial t}+u \frac{\partial u}{\partial x_{1}}-\left(\int_{-h}^{x_{2}} \frac{\partial}{\partial x_{1}} u\left(x_{1}, \zeta, t\right) \mathrm{d} \zeta\right) \frac{\partial u}{\partial x_{2}}-v\right. \\
& \left.+\frac{\partial p_{s}}{\partial x_{1}}-\left(\int_{x_{2}}^{0} \frac{\partial}{\partial x_{1}} T\left(x_{1}, \zeta, t\right) \mathrm{d} \zeta\right)-\frac{\partial^{2}}{\partial x_{1}^{2}} u\right\} \mathrm{d} A \mathrm{~d} \eta \\
& =\frac{1}{2} \int_{0}^{t} \int_{\Omega}\left(\frac{\partial^{2} S_{1}}{\partial x_{2}^{2}}\right)^{2} \mathrm{~d} A \mathrm{~d} \eta+\frac{1}{2} \int_{0}^{t} \int_{\Omega}\left|\nabla \frac{\partial u}{\partial x_{2}}\right|^{2} \mathrm{~d} A \mathrm{~d} \eta \\
& +\int_{\Omega} \frac{\partial S_{1}}{\partial x_{2}} \frac{\partial u}{\partial x_{2}} \mathrm{~d} A+\int_{0}^{t} \int_{\Omega} \frac{\partial\left(S_{1}\right)_{t}}{\partial x_{2}} \frac{\partial u}{\partial x_{2}} \mathrm{~d} A \mathrm{~d} \eta+\int_{0}^{t} \int_{\Omega} \frac{\partial S_{1}}{\partial x_{2}} \frac{\partial v}{\partial x_{2}} \mathrm{~d} A \mathrm{~d} \eta \\
& +\int_{0}^{t} \int_{\Omega} \frac{\partial S_{1}}{\partial x_{2}}\left[u \frac{\partial^{2} u}{\partial x_{1} \partial x_{2}}-\left(\int_{-h}^{x_{2}} \frac{\partial}{\partial x_{1}} u\left(x_{1}, \zeta, \eta\right) \mathrm{d} \zeta\right) \frac{\partial^{2} u}{\partial x_{2}^{2}}\right] \mathrm{d} A \mathrm{~d} \eta \\
& +\int_{0}^{t} \int_{\Omega} \frac{\partial S_{1}}{\partial x_{2}} \frac{\partial T}{\partial x_{1}} \mathrm{~d} A \mathrm{~d} \eta+\int_{0}^{t} \int_{\Omega} \frac{\partial^{2} S_{1}}{\partial x_{1} \partial x_{2}} \frac{\partial^{2} u}{\partial x_{1} \partial x_{2}} \mathrm{~d} A \mathrm{~d} \eta .
\end{aligned}
$$


8

Discrete Dynamics in Nature and Society

By the Cauchy-Schwarz inequality and Lemmas 4 and 5, the following is obtained:

$$
\begin{aligned}
& \int_{\Omega} \frac{\partial S_{1}}{\partial x_{2}} \frac{\partial u}{\partial x_{2}} \mathrm{~d} A \leq \int_{\Omega}\left(\frac{\partial S_{1}}{\partial x_{2}}\right)^{2} \mathrm{~d} A \mathrm{~d} \eta+\frac{1}{4} \int_{\Omega}\left(\frac{\partial u}{\partial x_{2}}\right)^{2} \mathrm{~d} A \\
& \int_{0}^{t} \int_{\Omega} \frac{\partial\left(S_{1}\right)_{t}}{\partial x_{2}} \frac{\partial u}{\partial x_{2}} \mathrm{~d} A \mathrm{~d} \eta \leq \sqrt{\int_{0}^{t} \int_{\Omega}\left(\frac{\partial\left(S_{1}\right)_{t}}{\partial x_{2}}\right)^{2} \mathrm{~d} A \mathrm{~d} \eta \int_{0}^{t} \int_{\Omega}\left(\frac{\partial u}{\partial x_{2}}\right)^{2} \mathrm{~d} A \mathrm{~d} \eta} \\
& \leq \sqrt{F_{3}(t) \int_{0}^{t} \int_{\Omega}\left(\frac{\partial\left(S_{1}\right)_{t}}{\partial x_{2}}\right)^{2} \mathrm{~d} A \mathrm{~d} \eta} \\
& \int_{0}^{t} \int_{\Omega} \frac{\partial S_{1}}{\partial x_{2}} \frac{\partial v}{\partial x_{2}} \mathrm{~d} A \mathrm{~d} \eta \leq \sqrt{\int_{0}^{t} \int_{\Omega}\left(\frac{\partial S_{1}}{\partial x_{2}}\right)^{2} \mathrm{~d} A \mathrm{~d} \eta \int_{0}^{t} \int_{\Omega}\left(\frac{\partial v}{\partial x_{2}}\right)^{2} \mathrm{~d} A \mathrm{~d} \eta} \\
& \leq \sqrt{F_{3}(t) \int_{0}^{t} \int_{\Omega}\left(\frac{\partial S_{1}}{\partial x_{2}}\right)^{2} \mathrm{~d} A \mathrm{~d} \eta}, \\
& \int_{0}^{t} \int_{\Omega} \frac{\partial S_{1}}{\partial x_{2}} \frac{\partial T}{\partial x_{1}} \mathrm{~d} A \mathrm{~d} \eta \leq \sqrt{\int_{0}^{t} \int_{\Omega}\left(\frac{\partial S_{1}}{\partial x_{2}}\right)^{2} \mathrm{~d} A \mathrm{~d} \eta \int_{0}^{t} \int_{\Omega}\left(\frac{\partial T}{\partial x_{1}}\right)^{2} \mathrm{~d} A \mathrm{~d} \eta} \\
& \leq \sqrt{F_{1}(t) \int_{0}^{t} \int_{\Omega}\left(\frac{\partial S_{1}}{\partial x_{2}}\right)^{2} \mathrm{~d} A \mathrm{~d} \eta}, \\
& \int_{0}^{t} \int_{\Omega} \frac{\partial^{2} S_{1}}{\partial x_{1} \partial x_{2}} \frac{\partial^{2} u}{\partial x_{1} \partial x_{2}} \mathrm{~d} A \mathrm{~d} \eta \leq 2 \int_{0}^{t} \int_{\Omega}\left(\frac{\partial^{2} S_{1}}{\partial x_{1} \partial x_{2}}\right)^{2} \mathrm{~d} A \mathrm{~d} \eta+\frac{1}{8} \int_{0}^{t} \int_{\Omega}\left|\nabla \frac{\partial u}{\partial x_{2}}\right|^{2} \mathrm{~d} A \mathrm{~d} \eta
\end{aligned}
$$


Discrete Dynamics in Nature and Society

9

and by (13) with $\delta=1$ and Lemma 5 ,

$$
\begin{aligned}
& \int_{0}^{t} \int_{\Omega} \frac{\partial S_{1}}{\partial x_{2}}\left[u \frac{\partial^{2} u}{\partial x_{1} x_{2}}-\left(\int_{-h}^{x_{2}} \frac{\partial}{\partial x_{1}} u\left(x_{1}, \zeta, \eta\right) \mathrm{d} \zeta\right) \frac{\partial^{2} u}{\partial x_{2}^{2}}\right] \mathrm{d} A \mathrm{~d} \eta \\
& =-\int_{0}^{t} \int_{\Omega} \frac{\partial^{2} S_{1}}{\partial x_{1} \partial x_{2}} u \frac{\partial u}{\partial x_{2}} \mathrm{~d} A \mathrm{~d} \eta+\int_{0}^{t} \int_{\Omega} \frac{\partial^{2} S_{1}}{\partial x_{2}^{2}}\left(\int_{-h}^{x_{2}} \frac{\partial}{\partial x_{1}} u\left(x_{1}, \zeta, \eta\right) d \zeta\right) \frac{\partial u}{\partial x_{2}} \mathrm{~d} A \mathrm{~d} \eta \\
& \leq\left[\int_{0}^{t} \int_{\Omega}\left(\frac{\partial u}{\partial x_{2}}\right)^{2} \mathrm{~d} A\right]^{1 / 2}\left[\int_{0}^{t}\left(\int_{\Omega} u^{4} \mathrm{~d} A\right)^{1 / 2}\left(\int_{\Omega}\left|\frac{\partial^{2} S_{1}}{\partial x_{1} \partial x_{2}}\right|^{4} \mathrm{~d} A\right)^{1 / 2} \mathrm{~d} \eta\right]^{1 / 2} \\
& \left.+\left[\int_{0}^{t} \int_{\Omega}\left(\int_{-h}^{x_{2}} \frac{\partial}{\partial x_{1}} u\left(x_{1}, \zeta, \eta\right) \mathrm{d} \zeta\right)^{2} \mathrm{~d} A\right]^{1 / 2}\left[\int_{0}^{t}\left(\int_{\Omega} \frac{\partial^{2} S_{1}}{\partial x_{2}^{2}}\right)^{4} \mathrm{~d} A\right)^{1 / 2}\left(\int_{\Omega}\left|\frac{\partial u}{\partial x_{2}}\right|^{4} \mathrm{~d} A\right)^{1 / 2} \mathrm{~d} \eta\right]^{1 / 2} \\
& \leq \max _{t}\left\{\sqrt[4]{\int_{\Omega}\left|\frac{\partial^{2} S_{1}}{\partial x_{1} \partial x_{2}}\right|^{4} \mathrm{~d} A}\right\} \sqrt{C F_{3}(t)}\left[\int_{0}^{t}\left(\int_{\Omega} u^{2} d A+\int_{\Omega}|\nabla u|^{2} \mathrm{~d} A\right) \mathrm{d} \eta\right]^{1 / 2}
\end{aligned}
$$

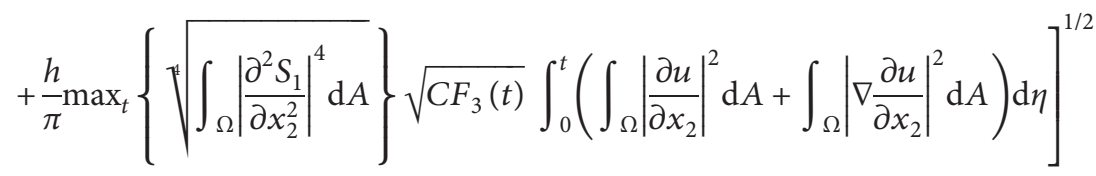

$$
\begin{aligned}
& \leq \max _{t}\left\{\sqrt[4]{\int_{\Omega}\left|\frac{\partial^{2} S_{1}}{\partial x_{1} \partial x_{2}}\right|^{4} \mathrm{~d} A}\right\} \sqrt{C F_{3}(t)}\left[\int_{0}^{t} F_{2}(\eta) \mathrm{d} \eta+F_{3}(t)\right]^{1 / 2}
\end{aligned}
$$

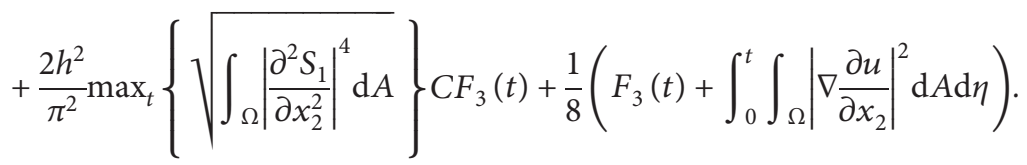

Inserting the above two inequalities into (61), one obtains

$$
\int_{0}^{t} \int_{0}^{1} \frac{\partial u}{\partial x_{2}} \frac{\partial^{2} u}{\partial x_{2}^{2}} \mathrm{~d} x_{1} \mathrm{~d} \eta \leq \frac{1}{4} \int_{\Omega}\left(\frac{\partial u}{\partial x_{2}}\right)^{2} \mathrm{~d} A+\frac{3}{4} \int_{0}^{t} \int_{\Omega}\left|\nabla \frac{\partial u}{\partial x_{2}}\right|^{2} \mathrm{~d} A \mathrm{~d} \eta+a_{4}(t)
$$


where

$$
\begin{aligned}
& a_{4}(t)=\frac{1}{2} \int_{0}^{t} \int_{\Omega}\left(\frac{\partial^{2} S_{1}}{\partial x_{2}^{2}}\right)^{2} \mathrm{~d} A \mathrm{~d} \eta+\int_{\Omega}\left(\frac{\partial S_{1}}{\partial x_{2}}\right)^{2} \mathrm{~d} A \mathrm{~d} \eta+\sqrt{F_{3}(t) \int_{0}^{t} \int_{\Omega}\left(\frac{\partial\left(S_{1}\right)_{t}}{\partial x_{2}}\right)^{2} \mathrm{~d} A \mathrm{~d} \eta} \\
& +\sqrt{F_{3}(t) \int_{0}^{t} \int_{\Omega}\left(\frac{\partial S_{1}}{\partial x_{2}}\right)^{2} \mathrm{~d} A \mathrm{~d} \eta}+\sqrt{F_{1}(t) \int_{0}^{t} \int_{\Omega}\left(\frac{\partial S_{1}}{\partial x_{2}}\right)^{2} \mathrm{~d} A \mathrm{~d} \eta}+2 \int_{0}^{t} \int_{\Omega}\left(\frac{\partial^{2} S_{1}}{\partial x_{1} \partial x_{2}}\right)^{2} \mathrm{~d} A \mathrm{~d} \eta \\
& +\max _{t}\left\{\sqrt[4]{\int_{\Omega}\left|\frac{\partial^{2} S_{1}}{\partial x_{1} \partial x_{2}}\right|^{4} \mathrm{~d} A}\right\} \sqrt{C F_{3}(t)}\left[\int_{0}^{t} F_{2}(\eta) \mathrm{d} \eta+F_{3}(t)\right]^{1 / 2}
\end{aligned}
$$

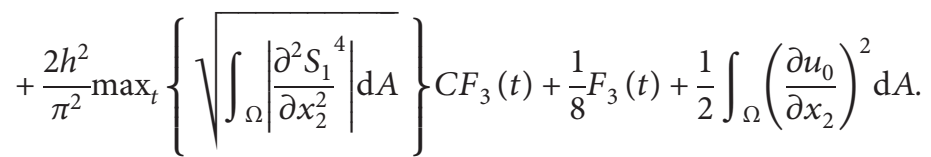

Combining the inequalities (59), (60), and (64), the following is obtained:

$$
\begin{gathered}
\int_{\Omega}\left(\frac{\partial u}{\partial x_{2}}\right)^{2} \mathrm{~d} A+\int_{0}^{t} \int_{\Omega}\left|\nabla \frac{\partial u}{\partial x_{2}}\right|^{2} \mathrm{~d} A \mathrm{~d} \eta \\
\leq 4 \int_{0}^{t} \int_{\Omega} \frac{\partial v}{\partial x_{2}} \frac{\partial u}{\partial x_{2}} \mathrm{~d} A \mathrm{~d} \eta+a_{5}(t),
\end{gathered}
$$

where $a_{5}(t)=4 a_{4}(t)+4 \sqrt{F_{1}(t) F_{3}(t)}$. Similar to (58) and (59), by $(2.4)_{2}$, one has

$$
\begin{aligned}
& \frac{1}{2} \int_{\Omega}\left(\frac{\partial v}{\partial x_{2}}\right)^{2} \mathrm{~d} A+\int_{\Omega}\left|\nabla \frac{\partial v}{\partial x_{2}}\right|^{2} \mathrm{~d} A \mathrm{~d} \eta= \int_{0}^{t} \int_{0}^{1} \frac{\partial v}{\partial x_{2}} \frac{\partial^{2} v}{\partial x_{2}^{2}} \mathrm{~d} x_{1} \mathrm{~d} \eta-\int_{\Omega} \frac{\partial v}{\partial x_{2}} \frac{\partial u}{\partial x_{2}} \mathrm{~d} A \mathrm{~d} \eta \\
&+\frac{1}{2} \int_{\Omega}\left(\frac{\partial v_{0}}{\partial x_{2}}\right)^{2} \mathrm{~d} A+\int_{\Omega}\left(\frac{\partial u}{\partial x_{2}} \frac{\partial v}{\partial x_{1}}-\frac{\partial u}{\partial x_{1}} \frac{\partial v}{\partial x_{2}}\right. \\
&-\int_{\Omega}\left[u \frac{\partial^{2} v}{\partial x_{1} x_{2}}-\left(\int_{-h}^{x_{2}} \frac{\partial}{\partial x_{1}} u\left(x_{1}, \zeta, t\right) \mathrm{d} \zeta\right)\right. \\
& \int_{\Omega}\left(\frac{\partial v}{\partial x_{2}}\right)^{2} \mathrm{~d} A+\int_{0}^{t} \int_{\Omega}\left|\nabla \frac{\partial v}{\partial x_{2}}\right|^{2} \mathrm{~d} A \mathrm{~d} \eta \quad \text { (68) } \\
& \leq-4 \int_{0}^{t} \int_{\Omega} \frac{\partial v}{\partial x_{2}} \frac{\partial u}{\partial x_{2}} \mathrm{~d} A \mathrm{~d} \eta+\int_{0}^{t} \int_{\Omega}\left|\nabla \frac{\partial v}{\partial x_{2}}\right|_{\Omega}^{2} \mathrm{~d} A \mathrm{~d} \eta \leq
\end{aligned}
$$$$
+\frac{1}{2} \int_{\Omega}\left(\frac{\partial v_{0}}{\partial x_{2}}\right)^{2} \mathrm{~d} A+\int_{\Omega}\left(\frac{\partial u}{\partial x_{2}} \frac{\partial v}{\partial x_{1}}-\frac{\partial u}{\partial x_{1}} \frac{\partial v}{\partial x_{2}}\right) \frac{\partial v}{\partial x_{2}} \mathrm{~d} A
$$$$
-\int_{\Omega}\left[u \frac{\partial^{2} v}{\partial x_{1} x_{2}}-\left(\int_{-h}^{x_{2}} \frac{\partial}{\partial x_{1}} u\left(x_{1}, \zeta, t\right) \mathrm{d} \zeta\right) \frac{\partial^{2} v}{\partial x_{2}^{2}}\right] \frac{\partial v}{\partial x_{2}} \mathrm{~d} A \mathrm{~d} \eta .
$$

In a similar way,

for some computable positive function $a_{6}(t)$. Combining (66) and (67), one obtains

where $F_{5}(t)=a_{5}(t)+a_{6}(t)$. Using Lemma 3 with $\delta=1$, Lemma 5, and (69), 


$$
\begin{aligned}
& \int_{0}^{t} \int_{\Omega}\left(\frac{\partial u}{\partial x_{2}}\right)^{4} \mathrm{~d} A \mathrm{~d} \eta+\int_{0}^{t} \int_{\Omega}\left(\frac{\partial v}{\partial x_{2}}\right)^{4} \mathrm{~d} A \mathrm{~d} \eta \leq C\left[\int_{0}^{t} \int_{\Omega}\left(\frac{\partial u}{\partial x_{2}}\right)^{2} \mathrm{~d} A \mathrm{~d} \eta\right. \\
& \left.\quad+\int_{0}^{t} \int_{\Omega}\left(\frac{\partial v}{\partial x_{2}}\right)^{2} \mathrm{~d} A \mathrm{~d} \eta+\int_{0}^{t} \int_{\Omega}\left|\nabla \frac{\partial u}{\partial x_{2}}\right|^{2} \mathrm{~d} A \mathrm{~d} \eta+\int_{0}^{t} \int_{\Omega}\left|\nabla \frac{\partial v}{\partial x_{2}}\right|^{2} \mathrm{~d} A \mathrm{~d} \eta\right]^{2} \\
& \leq C\left(F_{3}(t)+F_{5}(t)\right)^{2} \doteq F_{6}(t) .
\end{aligned}
$$

\section{Convergence Results on \\ Boundary Conditions}

$$
\widetilde{u}=u-u^{*}, \widetilde{v}=v-v^{*}, \widetilde{T}=T-T^{*}, \pi_{s}=p_{s}-p_{s}^{*},
$$

then $(\widetilde{u}, \widetilde{v}, \widetilde{T})$ satisfies

Supposing $\left(u^{*}, v^{*}, T^{*}\right)$ also be the solutions of (9)-(11) with the boundary conditions $g_{1}\left(x_{1}, t\right)=g_{2}\left(x_{1}, t\right)=T^{*}\left(x_{1}, t\right)=0$. Let

$$
\begin{array}{r}
\frac{\partial \widetilde{u}}{\partial t}+\widetilde{u} \frac{\partial u}{\partial x_{1}}-\left(\int_{-h}^{x_{2}} \frac{\partial}{\partial x_{1}} \widetilde{u}\left(x_{1}, \zeta, t\right) \mathrm{d} \zeta\right) \frac{\partial u}{\partial x_{2}}+u^{*} \frac{\partial \widetilde{u}}{\partial x_{1}}-\left(\int_{-h}^{x_{2}} \frac{\partial}{\partial x_{1}} u^{*}\left(x_{1}, \zeta, t\right) \mathrm{d} \zeta\right) \frac{\partial \widetilde{u}}{\partial x_{2}} \\
-\widetilde{v}+\frac{\partial \pi_{s}}{\partial x_{1}}-\left(\int_{x_{2}}^{0} \frac{\partial}{\partial x_{1}} \widetilde{T}\left(x_{1}, \zeta, t\right) \mathrm{d} \zeta\right)=\Delta \widetilde{u}, \\
\frac{\partial \widetilde{v}}{\partial t}+\widetilde{u} \frac{\partial v}{\partial x_{1}}-\left(\int_{-h}^{x_{2}} \frac{\partial}{\partial x_{1}} \widetilde{u}\left(x_{1}, \zeta, t\right) \mathrm{d} \zeta\right) \frac{\partial v}{\partial x_{2}}+u^{*} \frac{\partial \widetilde{v}}{\partial x_{1}}-\left(\int_{-h}^{x_{2}} \frac{\partial}{\partial x_{1}} u^{*}\left(x_{1}, \zeta, t\right) \mathrm{d} \zeta\right) \frac{\partial \widetilde{v}}{\partial x_{2}}-\widetilde{u}=\Delta \widetilde{v}, \\
\frac{\partial \widetilde{T}}{\partial t}+\widetilde{u} \frac{\partial T}{\partial x_{1}}-\left(\int_{-h}^{x_{2}} \frac{\partial}{\partial x_{1}} \widetilde{u}\left(x_{1}, \zeta, t\right) \mathrm{d} \zeta\right) \frac{\partial T}{\partial x_{2}}+u^{*} \frac{\partial \widetilde{T}}{\partial x_{1}}-\left(\int_{-h}^{x_{2}} \frac{\partial}{\partial x_{1}} u^{*}\left(x_{1}, \zeta, t\right) \mathrm{d} \zeta\right) \frac{\partial \widetilde{T}}{\partial x_{2}}=\Delta \widetilde{T},
\end{array}
$$

with the following boundary conditions:

$$
\begin{aligned}
& \left.\frac{\partial \widetilde{u}}{\partial x_{2}}\right|_{x_{2}=0}=\alpha_{1} g_{1}\left(x_{1}, t\right), \\
& \left.\frac{\partial \widetilde{v}}{\partial x_{2}}\right|_{x_{2}=0}=\alpha_{2} g_{2}\left(x_{1}, t\right), \\
& \left.\frac{\partial \widetilde{u}}{\partial x_{2}}\right|_{x_{2}=-h}=\left.\frac{\partial \widetilde{v}}{\partial x_{2}}\right|_{x_{2}=-h}=0 \\
& \left.\frac{\left.(\widetilde{u}, \widetilde{v})\right|_{\Gamma_{s}}=0}{\partial \widetilde{T}}\right|_{x_{2}=0}=-\beta T^{*}\left(x_{1}, t\right), \\
& \left.\frac{\partial \widetilde{T}}{\partial x_{2}}\right|_{x_{2}=-h}=0 \\
& \left.\frac{\partial \widetilde{T}}{\partial x_{1}}\right|_{\Gamma_{s}}=0
\end{aligned}
$$

and the initial conditions:

$$
\left.(\widetilde{u}, \widetilde{v}, \widetilde{T})\right|_{t=0}=(0,0,0) .
$$

The following theorem is obtained.

Theorem 1. Let $(\widetilde{\mathcal{u}}, \widetilde{v}, \widetilde{T})$ be the solutions of (71)-(73) with $g_{1}, g_{2}, T^{*} \in H^{1}(\Omega), T^{*}, T_{0} \in L_{\infty}(\Omega)$, and $u_{0}, v_{0} \in L_{2}(\Omega)$. Then, $(\widetilde{u}, \widetilde{v}, \widetilde{T})$ satisfies the inequality

$$
\begin{aligned}
& \frac{3 h^{2}}{\pi^{2}} T_{m}^{2} \int_{\Omega} \widetilde{u}^{2} \mathrm{~d} A+\frac{3 h^{2}}{\pi^{2}} T_{m}^{2} \int_{\Omega} \widetilde{v}^{2} \mathrm{~d} A+\frac{h^{2}}{\pi^{2}} T_{m}^{2} \int_{0}^{t} \int_{\Omega}|\nabla \widetilde{u}|^{2} \mathrm{~d} A \mathrm{~d} \eta \\
& \quad+\frac{3 h^{2}}{\pi^{2}} T_{m}^{2} \int_{0}^{t} \int_{\Omega}|\nabla \widetilde{v}|^{2} \mathrm{~d} A \mathrm{~d} \eta+\int_{\Omega} \widetilde{T}^{2} \mathrm{~d} A \\
& \quad+\frac{1}{2} \int_{0}^{t} \int_{\Omega}|\nabla \widetilde{T}|^{2} \mathrm{~d} A \mathrm{~d} \eta \\
& \leq \int_{0}^{t} \int_{0}^{1}\left[\frac{3 h^{2} \alpha_{1}}{\pi^{2}} T_{m}^{2} g_{1}^{2}\left(x_{1}, \eta\right)+\frac{3 h^{2} \alpha_{2}}{\pi^{2}} T_{m}^{2} g_{2}^{2}\left(x_{1}, \eta\right)\right. \\
& \left.\quad+\beta\left(T^{*}\right)^{2}\left(x_{1}, \eta\right)\right] \mathrm{d} x_{1} \mathrm{~d} \eta \\
& \quad+b(t) \int_{0}^{t} \int_{0}^{s} \int_{0}^{1} e \int_{s}^{t} b(\eta) \mathrm{d} \eta\left[\frac{3 h^{2} \alpha_{1}}{\pi^{2}} T_{m}^{2} g_{1}^{2}\left(x_{1}, \eta\right)\right. \\
& \left.\quad+\frac{3 h^{2} \alpha_{2}}{\pi^{2}} T_{m}^{2} g_{2}^{2}\left(x_{1}, \eta\right)+\beta\left(T^{*}\right)^{2}\left(x_{1}, \eta\right)\right] \mathrm{d} x_{1} \mathrm{~d} \eta \mathrm{d} s,
\end{aligned}
$$


which are the convergence results on boundary conditions $g_{1}, g_{2}, T^{*}$.
Proof. Now taking the inner product of equation $(72)_{1}$ with $\tilde{u}$, in $L_{2}(\Omega)$, the following is obtained:

$$
\begin{aligned}
\frac{1}{2} \int_{\Omega} \widetilde{u}^{2} \mathrm{~d} A+\int_{0}^{t} \int_{\Omega}|\nabla \widetilde{u}|^{2} \mathrm{~d} A \mathrm{~d} \eta= & \int_{0}^{t} \int_{\Omega} \widetilde{u} \widetilde{v} \mathrm{~d} \mathrm{~d} \eta-\int_{0}^{t} \int_{\Omega} \frac{\partial \pi_{s}}{\partial x_{1}} \widetilde{u} \mathrm{~d} A \mathrm{~d} \eta \\
& +\int_{0}^{t} \int_{0}^{1} \frac{\partial \widetilde{u}}{\partial x_{2}} \widetilde{u} \mathrm{~d} x_{1} \mathrm{~d} \eta+\int_{0}^{t} \int_{\Omega}\left(\int_{x_{2}}^{0} \frac{\partial}{\partial x_{1}} \widetilde{T}\left(x_{1}, \zeta, \eta\right) \mathrm{d} \zeta\right) \widetilde{u} \mathrm{~d} A \mathrm{~d} \eta \\
& -\int_{0}^{t} \int_{\Omega}\left[\widetilde{u} \frac{\partial u}{\partial x_{1}}-\left(\int_{-h}^{x_{2}} \frac{\partial}{\partial x_{1}} \widetilde{u}\left(x_{1}, \zeta, \eta\right) \mathrm{d} \zeta\right) \frac{\partial u}{\partial x_{2}}\right] \widetilde{u} \mathrm{~d} A \mathrm{~d} \eta .
\end{aligned}
$$

By the Hölder inequality, Lemmas 1, 5, and 7, and the A-G mean inequality, the following is obtained:

$$
\begin{aligned}
& -\int_{0}^{t} \int_{\Omega}\left[\widetilde{u} \frac{\partial u}{\partial x_{1}}-\left(\int_{-h}^{x_{2}} \frac{\partial}{\partial x_{1}} \widetilde{u}\left(x_{1}, \zeta, \eta\right) \mathrm{d} \zeta\right) \frac{\partial u}{\partial x_{2}}\right] \widetilde{u} \mathrm{~d} A \mathrm{~d} \eta \leq\left[\int_{0}^{t} \int_{\Omega}\left(\frac{\partial u}{\partial x_{1}}\right)^{2} \mathrm{~d} A\right]^{1 / 2}\left[\int_{0}^{t} \int_{\Omega} \widetilde{u}^{4} \mathrm{~d} A \mathrm{~d} \eta\right]^{1 / 2} \\
& +\left[\int_{0}^{t} \int_{\Omega}\left(\int_{-h}^{x_{2}} \frac{\partial}{\partial x_{1}} \widetilde{u}\left(x_{1}, \zeta, \eta\right) \mathrm{d} \zeta\right)^{2} \mathrm{~d} A \mathrm{~d} \eta\right]^{1 / 2}\left[\int_{0}^{t} \int_{\Omega}\left(\frac{\partial u}{\partial x_{2}}\right)^{4} \mathrm{~d} A \mathrm{~d} \eta\right]^{1 / 2}\left[\int_{0}^{t} \int_{\Omega} \widetilde{u}^{4} \mathrm{~d} A \mathrm{~d} \eta\right]^{1 / 4} \\
& \leq \sqrt{F_{3}(t)} C\left[\int_{0}^{t} \int_{\Omega} \widetilde{u}^{2} \mathrm{~d} A \mathrm{~d} \eta+\delta_{1} \int_{0}^{t} \int_{\Omega}|\nabla \widetilde{u}|^{2} \mathrm{~d} A \mathrm{~d} \eta\right] \\
& +\frac{\sqrt{C} h}{\pi} \sqrt[4]{F_{6}(t)}\left[\int_{0}^{t} \int_{\Omega}\left(\frac{\partial \widetilde{u}}{\partial x_{1}}\right)^{2} \mathrm{~d} A \mathrm{~d} \eta\right]^{1 / 2}\left[\int_{0}^{t} \int_{\Omega} \tilde{u}^{2} d A d \eta+\delta_{1} \int_{0}^{t} \int_{\Omega}|\nabla \widetilde{u}|^{2} \mathrm{~d} A \mathrm{~d} \eta\right]^{1 / 2} \\
& \leq \sqrt{F_{3}(t)} C\left[\int_{0}^{t} \int_{\Omega} \tilde{u}^{2} \mathrm{~d} A \mathrm{~d} \eta+\delta_{1} \int_{0}^{t} \int_{\Omega}|\nabla \tilde{u}|^{2} \mathrm{~d} A \mathrm{~d} \eta\right] \\
& +\frac{\sqrt{C} h}{\pi} \sqrt[4]{F_{6}(t)}\left[\int_{0}^{t} \int_{\Omega}\left(\frac{\partial \tilde{u}}{\partial x_{1}}\right)^{2} \mathrm{~d} A \mathrm{~d} \eta\right]^{1 / 2}\left[\int_{0}^{t} \int_{\Omega} \widetilde{u}^{2} \mathrm{~d} A \mathrm{~d} \eta\right]^{1 / 2} \\
& +\frac{\sqrt{C \delta_{1}} h}{\pi} \sqrt[4]{F_{6}(t)}\left[\int_{0}^{t} \int_{\Omega}\left(\frac{\partial \widetilde{u}}{\partial x_{1}}\right)^{2} \mathrm{~d} A \mathrm{~d} \eta\right]^{1 / 2}\left[\int_{0}^{t} \int_{\Omega}|\nabla \widetilde{u}|^{2} \mathrm{~d} A \mathrm{~d} \eta\right]^{1 / 2} \\
& \leq\left[\sqrt{F_{3}(t)} C+\frac{C h^{2}}{4 \delta_{2} \pi^{2}} \sqrt{F_{6}(t)}\right] \int_{0}^{t} \int_{\Omega} \tilde{u}^{2} \mathrm{~d} A \mathrm{~d} \eta \\
& +\left[\sqrt{F_{3}(t)} C \delta_{1}+\delta_{2}+\frac{\sqrt{C \delta_{1}} h}{\pi} \sqrt[4]{F_{6}(t)}\right] \int_{0}^{t} \int_{\Omega}|\nabla \widetilde{u}|^{2} \mathrm{~d} A \mathrm{~d} \eta,
\end{aligned}
$$


for arbitrary positive constants $\delta_{1}$ and $\delta_{2}$. By the Cauchy-Schwarz inequality again

$$
\begin{aligned}
\int_{0}^{t} \int_{\Omega}\left(\int_{x_{2}}^{0} \frac{\partial}{\partial x_{1}} \widetilde{T}\left(x_{1}, \zeta, \eta\right) \mathrm{d} \zeta\right) \widetilde{u} \mathrm{~d} A \mathrm{~d} \eta & \leq \frac{\pi^{2}}{12 h^{2} T_{m}^{2}} \int_{0}^{t} \int_{\Omega}\left(\frac{\partial}{\partial x_{1}} \widetilde{T}\right)^{2} \mathrm{~d} A \mathrm{~d} \eta+\frac{3 h^{4}}{\pi^{2}} T_{m}^{2} \int_{0}^{t} \int_{\Omega} \tilde{u}^{2} \mathrm{~d} A \mathrm{~d} \eta \\
\int_{0}^{t} \int_{0}^{1} \frac{\partial \widetilde{u}}{\partial x_{2}} \tilde{u} \mathrm{~d} x_{1} \mathrm{~d} \eta & =\alpha_{1} \int_{0}^{t} \int_{0}^{1} g_{1}\left(x_{1}, t\right) \widetilde{u}\left(x_{1}, 0, t\right) \mathrm{d} x_{1} \mathrm{~d} \eta \\
& \leq \frac{\alpha_{1}}{2} \int_{0}^{t} \int_{0}^{1} g_{1}^{2}\left(x_{1}, t\right) \mathrm{d} x_{1} \mathrm{~d} \eta+\frac{\alpha_{1}}{2} \int_{0}^{t} \int_{0}^{1} \tilde{u}^{2}\left(x_{1}, 0, t\right) \mathrm{d} x_{1} \mathrm{~d} \eta .
\end{aligned}
$$

In view of (25), the following is obtained:

$$
\begin{aligned}
\int_{0}^{t} \int_{0}^{1} \frac{\partial \widetilde{u}}{\partial x_{2}} \tilde{u} \mathrm{~d} x_{1} \mathrm{~d} \eta \leq & \frac{\alpha_{1}}{2} \int_{0}^{t} \int_{0}^{1} g_{1}^{2}\left(x_{1}, t\right) \mathrm{d} x_{1} \mathrm{~d} \eta+\frac{m_{3} \alpha_{1}}{2} \int_{0}^{t} \int_{\Omega} \tilde{u}^{2} \mathrm{~d} A \mathrm{~d} \eta \\
& +\frac{m_{4} \alpha_{1}}{2} \int_{0}^{t} \int_{\Omega}|\widetilde{u}|\left|\frac{\partial \widetilde{u}}{\partial x_{1}}\right| \mathrm{d} A \mathrm{~d} \eta \\
\leq & \frac{\alpha_{1}}{2} \int_{0}^{t} \int_{0}^{1} g_{1}^{2} \mathrm{~d} x_{1} \mathrm{~d} \eta+\frac{\left(2 m_{3}+m_{4} \delta_{3}^{-1}\right) \alpha_{1}}{4} \int_{0}^{t} \int_{\Omega} \widetilde{u}^{2} \mathrm{~d} A \mathrm{~d} \eta \\
& +\frac{m_{4} \alpha_{1}}{4} \delta_{3} \int_{0}^{t} \int_{\Omega}|\nabla \widetilde{u}|^{2} \mathrm{~d} A \mathrm{~d} \eta .
\end{aligned}
$$

Inserting (77)-(80) into (76), one has

$$
\begin{aligned}
\frac{1}{2} \int_{\Omega} \widetilde{u}^{2} \mathrm{~d} A+\int_{0}^{t} \int_{\Omega}|\nabla \widetilde{u}|^{2} \mathrm{~d} A \mathrm{~d} \eta \leq & \int_{0}^{t} \int_{\Omega} \tilde{u} \widetilde{v} \mathrm{~d} A \mathrm{~d} \eta+\frac{\alpha_{1}}{2} \int_{0}^{t} \int_{0}^{1} g_{1}^{2} \mathrm{~d} x_{1} \mathrm{~d} \eta+\frac{\pi^{2}}{12 h^{2} T_{m}^{2}} \int_{0}^{t} \int_{\Omega}|\nabla \widetilde{T}|^{2} \mathrm{~d} A \mathrm{~d} \eta \\
& +\left[\sqrt{F_{3}(t)} C+\frac{C h^{2}}{4 \delta_{2} \pi^{2}} \sqrt{F_{6}(t)}+\frac{\left(2 m_{3}+m_{4} \delta_{3}^{-1}\right) \alpha_{1}}{4}+\frac{3 h^{4}}{\pi^{2}} T_{m}^{2}\right] \int_{0}^{t} \int_{\Omega} \tilde{u}^{2} \mathrm{~d} A \mathrm{~d} \eta \\
& +\left[\sqrt{F_{3}(t)} C \delta_{1}+\delta_{2}+\frac{\sqrt{C \delta_{1}} h}{\pi} \sqrt[4]{F_{6}(t)}+\frac{m_{4} \alpha_{1}}{4} \delta_{3}\right] \int_{0}^{t} \int_{\Omega}|\nabla \widetilde{u}|^{2} \mathrm{~d} A \mathrm{~d} \eta
\end{aligned}
$$

Now taking the inner product of the second equation of (72) with $\widetilde{v}$, the following is obtained:

$$
\begin{aligned}
\frac{1}{2} \int_{\Omega} \widetilde{v}^{2} \mathrm{~d} A+\int_{0}^{t} \int_{\Omega}|\nabla \widetilde{v}|^{2} \mathrm{~d} A \mathrm{~d} \eta= & -\int_{0}^{t} \int_{\Omega} \widetilde{u} \widetilde{v} \mathrm{~d} A \mathrm{~d} \eta+\int_{0}^{t} \int_{0}^{1} \frac{\partial \widetilde{v}}{\partial x_{2}} \widetilde{v} \mathrm{~d} x_{1} \mathrm{~d} \eta \\
& -\int_{0}^{t} \int_{\Omega}\left[\widetilde{u} \frac{\partial v}{\partial x_{1}}-\left(\int_{-h}^{x_{2}} \frac{\partial}{\partial x_{1}} \widetilde{u}\left(x_{1}, \zeta, \eta\right) \mathrm{d} \zeta\right) \frac{\partial v}{\partial x_{2}}\right] \widetilde{v} \mathrm{~d} A \mathrm{~d} \eta
\end{aligned}
$$


Computing as (80),

$$
\begin{gathered}
\int_{0}^{t} \int_{0}^{1} \frac{\partial \widetilde{v}}{\partial x_{2}} \widetilde{v} \mathrm{~d} x_{1} \mathrm{~d} \eta \leq \\
\frac{\alpha_{2}}{2} \int_{0}^{t} \int_{0}^{1} g_{2}^{2} \mathrm{~d} x_{1} \mathrm{~d} \eta+\frac{\left(2 m_{3}+m_{4} \delta_{4}^{-1}\right) \alpha_{2}}{4} \int_{0}^{t} \int_{\Omega} \widetilde{v}^{2} \mathrm{~d} A \mathrm{~d} \eta \\
+\frac{m_{4} \alpha_{2}}{4} \delta_{4} \int_{0}^{t} \int_{\Omega}|\nabla \widetilde{v}|^{2} \mathrm{~d} A \mathrm{~d} \eta .
\end{gathered}
$$

Computing as (77),

$$
\begin{aligned}
& \quad-\int_{0}^{t} \int_{\Omega}\left[\tilde{u} \frac{\partial v}{\partial x_{1}}-\left(\int_{-h}^{x_{2}} \frac{\partial}{\partial x_{1}} \tilde{u}\left(x_{1}, \zeta, \eta\right) \mathrm{d} \zeta\right) \frac{\partial v}{\partial x_{2}}\right] \widetilde{v} \mathrm{~d} A \mathrm{~d} \eta \\
& \leq \frac{C \sqrt{F_{3}(t)}}{2}\left(\int_{0}^{t} \int_{\Omega} \tilde{u}^{2} \mathrm{~d} A \mathrm{~d} \eta+\delta_{5} \int_{0}^{t} \int_{\Omega}|\nabla \widetilde{u}|^{2} \mathrm{~d} A \mathrm{~d} \eta\right) \\
& +\frac{C \sqrt{F_{3}(t)}}{2}\left(\int_{0}^{t} \int_{\Omega} \widetilde{v}^{2} \mathrm{~d} A \mathrm{~d} \eta+\delta_{5} \int_{0}^{t} \int_{\Omega}|\nabla \widetilde{v}|^{2} \mathrm{~d} A \mathrm{~d} \eta\right) \\
& +\frac{\sqrt{C F_{6}(t)} h}{\sqrt{\delta_{6}} \pi}\left(\int_{0}^{t} \int_{\Omega} \widetilde{v}^{2} \mathrm{~d} A \mathrm{~d} \eta+\delta_{6} \int_{0}^{t} \int_{\Omega}|\nabla \widetilde{u}|^{2} \mathrm{~d} A \mathrm{~d} \eta+\delta_{6} \int_{0}^{t} \int_{\Omega}|\nabla \widetilde{v}|^{2} \mathrm{~d} A \mathrm{~d} \eta\right),
\end{aligned}
$$

where $\delta_{5}$ and $\delta_{6}$ are the arbitrary constants. Inserting (83) and (84) into (82), the following is obtained:

$$
\begin{aligned}
\frac{1}{2} \int_{\Omega} \widetilde{v}^{2} \mathrm{~d} A+\int_{0}^{t} \int_{\Omega}|\nabla \widetilde{v}|^{2} \mathrm{~d} A \mathrm{~d} \eta \leq & \frac{\alpha_{2}}{2} \int_{0}^{t} \int_{0}^{1} g_{2}^{2} \mathrm{~d} x_{1} \mathrm{~d} \eta+\frac{C \sqrt{F_{3}(t)}}{2} \int_{0}^{t} \int_{\Omega} \tilde{u}^{2} \mathrm{~d} A \mathrm{~d} \eta \\
& +\left[\frac{\left(2 m_{3}+m_{4} \delta_{4}^{-1}\right) \alpha_{2}}{4}+\frac{C \sqrt{F_{3}(t)}}{2}+\frac{\sqrt{C F_{6}(t)} h}{\sqrt{\delta_{6}} \pi}\right] \int_{0}^{t} \int_{\Omega} \widetilde{v}^{2} \mathrm{~d} A \mathrm{~d} \eta \\
& +\left[\frac{m_{4} \alpha_{2}}{4} \delta_{4}+\frac{C \sqrt{F_{3}(t)}}{2} \delta_{5}+\frac{\sqrt{C \delta_{6} F_{6}(t)} h}{\pi}\right] \int_{0}^{t} \int_{\Omega}|\nabla \widetilde{v}|^{2} \mathrm{~d} A \mathrm{~d} \eta \\
& +\frac{\sqrt{C \delta_{6} F_{6}(t)} h}{\pi} \int_{0}^{t} \int_{\Omega}|\nabla \widetilde{u}|^{2} \mathrm{~d} A \mathrm{~d} \eta .
\end{aligned}
$$

(81) and (85) are combined and suitable $\delta_{i},(i=1,2, \ldots, 6)$ are chosen such that

$$
\begin{array}{r}
\sqrt{F_{3}(t)} C \delta_{1}+\delta_{2}+\frac{\sqrt{C \delta_{1}} h}{\pi} \sqrt[4]{F_{6}(t)}+\frac{m_{4} \alpha_{1}}{4} \delta_{3}+\frac{\sqrt{C \delta_{6} F_{6}(t)} h}{\pi}=\frac{1}{2}, \\
\frac{m_{4} \alpha_{2}}{4} \delta_{4}+\frac{C \sqrt{F_{3}(t)}}{2} \delta_{5}+\frac{\sqrt{C \delta_{6} F_{6}(t)} h}{\pi}=\frac{1}{2},
\end{array}
$$


to have

$$
\begin{array}{r}
\int_{\Omega} \widetilde{u}^{2} \mathrm{~d} A+\int_{\Omega} \widetilde{v}^{2} \mathrm{~d} A+\int_{0}^{t} \int_{\Omega}|\nabla \widetilde{u}|^{2} \mathrm{~d} A \mathrm{~d} \eta+\int_{0}^{t} \int_{\Omega}|\nabla \widetilde{v}|^{2} \mathrm{~d} A \mathrm{~d} \eta \leq \alpha_{1} \int_{0}^{t} \int_{0}^{1} g_{1}^{2} \mathrm{~d} x_{1} \mathrm{~d} \eta \\
+\alpha_{2} \int_{0}^{t} \int_{0}^{1} g_{2}^{2} \mathrm{~d} x_{1} \mathrm{~d} \eta+\frac{\pi^{2}}{6 h^{2} T_{m}^{2}} \int_{0}^{t} \int_{\Omega}|\nabla \widetilde{T}|^{2} \mathrm{~d} A \mathrm{~d} \eta \\
+\left[2 \sqrt{F_{3}(t)} C+\frac{C h^{2}}{2 \delta_{2} \pi^{2}} \sqrt{F_{6}(t)}+\frac{\left(2 m_{3}+m_{4} \delta_{3}^{-1}\right) \alpha_{1}}{2}+C \sqrt{F_{3}(t)}+\frac{6 h^{4}}{\pi^{2}} T_{m}^{2}\right] \int_{0}^{t} \int_{\Omega} \widetilde{u}^{2} \mathrm{~d} A \mathrm{~d} \eta \\
+\left[\frac{\left(2 m_{3}+m_{4} \delta_{4}^{-1}\right) \alpha_{2}}{2}+C \sqrt{F_{3}(t)}+\frac{2 \sqrt{C F_{6}(t)} h}{\sqrt{\delta_{6}} \pi}\right] \int_{0}^{t} \int_{\Omega} \widetilde{v}^{2} \mathrm{~d} A \mathrm{~d} \eta .
\end{array}
$$
have

The inner product of equation $(72)_{3}$ is taken with $\widetilde{T}$ to

$$
\begin{aligned}
\frac{1}{2} \int_{\Omega} \widetilde{T}^{2} \mathrm{~d} A+\int_{0}^{t} \int_{\Omega}|T|^{2} \mathrm{~d} A \mathrm{~d} \eta= & \int_{0}^{t} \int_{0}^{1} \frac{\partial \widetilde{T}}{\partial x_{2}} \widetilde{T} \mathrm{~d} x_{1} \mathrm{~d} \eta \\
& -\int_{0}^{t} \int_{\Omega}\left[\widetilde{u} \frac{\partial T}{\partial x_{1}}-\left(\int_{-h}^{x_{2}} \frac{\partial}{\partial x_{1}} \widetilde{u}\left(x_{1}, \zeta, \eta\right) \mathrm{d} \zeta\right) \frac{\partial T}{\partial x_{2}}\right] \widetilde{T} \mathrm{~d} A \mathrm{~d} \eta
\end{aligned}
$$

Similar to the computation in (79) and (80), the following is obtained:

$$
\begin{aligned}
\int_{0}^{t} \int_{0}^{1} \frac{\partial \widetilde{T}}{\partial x_{2}} \widetilde{T} \mathrm{~d} x_{1} \mathrm{~d} \eta \leq & \frac{\beta}{2} \int_{0}^{t} \int_{0}^{1}\left(T^{*}\right)^{2} \mathrm{~d} x_{1} \mathrm{~d} \eta \\
& +\frac{\left(2 m_{3}+m_{4} \delta_{7}^{-1}\right) \beta}{4} \int_{0}^{t} \int_{\Omega} \widetilde{T}^{2} \mathrm{~d} A \mathrm{~d} \eta \\
& +\frac{m_{4} \beta}{4} \delta_{7} \int_{0}^{t} \int_{\Omega}|\nabla \widetilde{T}|^{2} \mathrm{~d} A \mathrm{~d} \eta,
\end{aligned}
$$

for an arbitrary positive constant $\delta_{7}$. On integrating by parts, one has

$$
-\int_{0}^{t} \int_{\Omega}\left[u^{*} \frac{\partial \widetilde{T}}{\partial x_{1}}-\left(\int_{-h}^{x_{2}} \frac{\partial}{\partial x_{1}} u^{*}\left(x_{1}, \zeta, \eta\right) \mathrm{d} \zeta\right) \frac{\partial \widetilde{T}}{\partial x_{2}}\right] \widetilde{T} \mathrm{~d} A \mathrm{~d} \eta=0 .
$$

By the Hölder inequality and Lemmas 2 and 6, the following is obtained: 


$$
\begin{aligned}
& -\int_{0}^{t} \int_{\Omega}\left[\widetilde{u} \frac{\partial T}{\partial x_{1}}-\left(\int_{-h}^{x_{2}} \frac{\partial}{\partial x_{1}} \widetilde{u}\left(x_{1}, \zeta, \eta\right) \mathrm{d} \zeta\right) \frac{\partial T}{\partial x_{2}}\right] \widetilde{T} \mathrm{~d} A \mathrm{~d} \eta \\
= & \int_{0}^{t} \int_{\Omega} \widetilde{u} T \frac{\partial \widetilde{T}}{\partial x_{1}} \mathrm{~d} A \mathrm{~d} \eta-\int_{0}^{t} \int_{\Omega}\left(\int_{-h}^{x_{2}} \frac{\partial}{\partial x_{1}} \widetilde{u}\left(x_{1}, \zeta, \eta\right) \mathrm{d} \zeta\right) T \frac{\partial \widetilde{T}}{\partial x_{2}} \mathrm{~d} A \mathrm{~d} \eta \\
\leq & T_{m}\left(\int_{0}^{t} \int_{\Omega}\left(\frac{\partial \widetilde{T}}{\partial x_{1}}\right)^{2} \mathrm{~d} A \mathrm{~d} \eta\right)^{1 / 2}\left(\int_{0}^{t} \int_{\Omega} \widetilde{u}^{2} \mathrm{~d} A \mathrm{~d} \eta\right)^{1 / 2} \\
& +T_{m}\left(\int_{0}^{t} \int_{\Omega}\left(\int_{-h}^{x_{2}} \frac{\partial}{\partial x_{1}} \widetilde{u}\left(x_{1}, \zeta, \eta\right) \mathrm{d} \zeta\right)^{2} \mathrm{~d} A \mathrm{~d} \eta\right)^{1 / 2}\left(\int_{0}^{t} \int_{\Omega}\left(\frac{\partial \widetilde{T}}{\partial x_{2}}\right)^{2} \mathrm{~d} A \mathrm{~d} \eta\right)^{1 / 2} \\
\leq & T_{m}^{2} \int_{0}^{t} \int_{\Omega} \widetilde{u}^{2} \mathrm{~d} A \mathrm{~d} \eta+\frac{h^{2}}{\pi^{2}} T_{m}^{2} \int_{0}^{t} \int_{\Omega}|\nabla \widetilde{u}|^{2} \mathrm{~d} A \mathrm{~d} \eta+\frac{1}{4} \int_{0}^{t} \int_{\Omega}|\nabla \widetilde{T}|^{2} \mathrm{~d} A \mathrm{~d} \eta .
\end{aligned}
$$

Inserting (89)-(91) into (88) and choosing $\delta_{7}=\left(1 / m_{4} \beta\right)$, one gets

$$
\begin{aligned}
\int_{\Omega} \widetilde{T}^{2} \mathrm{~d} A+\int_{0}^{t} \int_{\Omega}|\nabla \widetilde{T}|^{2} \mathrm{~d} A \mathrm{~d} \eta \leq & \beta \int_{0}^{t} \int_{0}^{1}\left(T^{*}\right)^{2} \mathrm{~d} x_{1} \mathrm{~d} \eta+\frac{\left(2 m_{3}+m_{4} \delta_{7}^{-1}\right) \beta}{2} \int_{0}^{t} \int_{\Omega} \widetilde{T}^{2} \mathrm{~d} A \mathrm{~d} \eta \\
& +2 T_{m}^{2} \int_{0}^{t} \int_{\Omega} \widetilde{u}^{2} \mathrm{~d} A \mathrm{~d} \eta+\frac{2 h^{2}}{\pi^{2}} T_{m}^{2} \int_{0}^{t} \int_{\Omega}|\nabla \widetilde{u}|^{2} \mathrm{~d} A \mathrm{~d} \eta .
\end{aligned}
$$

Combining (87) and (92), the following is obtained:

$$
\begin{aligned}
& \frac{3 h^{2}}{\pi^{2}} T_{m}^{2} \int_{\Omega} \widetilde{u}^{2} \mathrm{~d} A+\frac{3 h^{2}}{\pi^{2}} T_{m}^{2} \int_{\Omega} \widetilde{v}^{2} \mathrm{~d} A+\frac{h^{2}}{\pi^{2}} T_{m}^{2} \int_{0}^{t} \int_{\Omega}|\nabla \widetilde{u}|^{2} \mathrm{~d} A \mathrm{~d} \eta \\
& \quad+\frac{3 h^{2}}{\pi^{2}} T_{m}^{2} \int_{0}^{t} \int_{\Omega}|\nabla \widetilde{v}|^{2} \mathrm{~d} A \mathrm{~d} \eta+\int_{\Omega} \widetilde{T}^{2} \mathrm{~d} A+\frac{1}{2} \int_{0}^{t} \int_{\Omega}|\nabla \widetilde{T}|^{2} \mathrm{~d} A \mathrm{~d} \eta \\
& \leq \int_{0}^{t} \int_{0}^{1}\left[\frac{3 h^{2} \alpha_{1}}{\pi^{2}} T_{m}^{2} g_{1}^{2}+\frac{3 h^{2} \alpha_{2}}{\pi^{2}} T_{m}^{2} g_{2}^{2}+\beta\left(T^{*}\right)^{2}\right] \mathrm{d} x_{1} \mathrm{~d} \eta \\
& \quad+b(t)\left(\frac{3 h^{2}}{\pi^{2}} T_{m}^{2} \int_{0}^{t} \int_{\Omega} \widetilde{u}^{2} \mathrm{~d} A \mathrm{~d} \eta+\frac{3 h^{2}}{\pi^{2}} T_{m}^{2} \int_{0}^{t} \int_{\Omega} \widetilde{v}^{2} \mathrm{~d} A \mathrm{~d} \eta+\int_{0}^{t} \int_{\Omega} \widetilde{T}^{2} \mathrm{~d} A \mathrm{~d} \eta\right),
\end{aligned}
$$


where

$$
\begin{aligned}
b(t)= & \max \left\{2 \sqrt{F_{3}(t)} C+\frac{C h^{2}}{2 \delta_{2} \pi^{2}} \sqrt{F_{6}(t)}+\frac{\left(2 m_{3}+m_{4} \delta_{3}^{-1}\right) \alpha_{1}}{2}+C \sqrt{F_{3}(t)}+\frac{6 h^{4}}{\pi^{2}} T_{m}^{2}+\frac{2 \pi^{2}}{3 h^{2}},\right. \\
& \left.\frac{\left(2 m_{3}+m_{4} \delta_{4}^{-1}\right) \alpha_{2}}{2}+C \sqrt{F_{3}(t)}+\frac{2 \sqrt{C F_{6}(t)} h}{\sqrt{\delta_{6}} \pi}, \frac{\left(2 m_{3}+m_{4} \delta_{7}^{-1}\right) \beta}{2}\right\} .
\end{aligned}
$$

Therefore, by the Gronwall inequality,

$$
\begin{aligned}
& \frac{3 h^{2}}{\pi^{2}} T_{m}^{2} \int_{0}^{t} \int_{\Omega} \widetilde{u}^{2} \mathrm{~d} A \mathrm{~d} \eta+\frac{3 h^{2}}{\pi^{2}} T_{m}^{2} \int_{0}^{t} \int_{\Omega} \widetilde{v}^{2} \mathrm{~d} A \mathrm{~d} \eta+\int_{0}^{t} \int_{\Omega} \widetilde{T}^{2} \mathrm{~d} A \mathrm{~d} \eta \\
& \quad \leq \int_{0}^{t} \int_{0}^{s} \int_{0}^{1} e \int_{s}^{t} b(\eta) \mathrm{d} \eta\left[\frac{3 h^{2} \alpha_{1}}{\pi^{2}} T_{m}^{2} g_{1}^{2}\left(x_{1}, \eta\right)+\frac{3 h^{2} \alpha_{2}}{\pi^{2}} T_{m}^{2} g_{2}^{2}\left(x_{1}, \eta\right)+\beta\left(T^{*}\right)^{2}\left(x_{1}, \eta\right)\right] \mathrm{d} x_{1} \mathrm{~d} \eta \mathrm{d} s .
\end{aligned}
$$

Then, returning to (93) it can be seen that

$$
\begin{aligned}
& \frac{3 h^{2}}{\pi^{2}} T_{m}^{2} \int_{\Omega} \widetilde{u}^{2} \mathrm{~d} A+\frac{3 h^{2}}{\pi^{2}} T_{m}^{2} \int_{\Omega} \widetilde{v}^{2} \mathrm{~d} A+\frac{h^{2}}{\pi^{2}} T_{m}^{2} \int_{0}^{t} \int_{\Omega}|\nabla \widetilde{u}|^{2} \mathrm{~d} A \mathrm{~d} \eta \\
& \quad+\frac{3 h^{2}}{\pi^{2}} T_{m}^{2} \int_{0}^{t} \int_{\Omega}|\nabla \widetilde{v}|^{2} \mathrm{~d} A \mathrm{~d} \eta+\int_{\Omega} \widetilde{T}^{2} \mathrm{~d} A+\frac{1}{2} \int_{0}^{t} \int_{\Omega}|\nabla \widetilde{T}|^{2} \mathrm{~d} A \mathrm{~d} \eta \\
& \leq \int_{0}^{t} \int_{0}^{1}\left[\frac{3 h^{2} \alpha_{1}}{\pi^{2}} T_{m}^{2} g_{1}^{2}\left(x_{1}, \eta\right)+\frac{3 h^{2} \alpha_{2}}{\pi^{2}} T_{m}^{2} g_{2}^{2}\left(x_{1}, \eta\right)+\beta\left(T^{*}\right)^{2}\left(x_{1}, \eta\right)\right] \mathrm{d} x_{1} \mathrm{~d} \eta \\
& \quad+b(t) \int_{0}^{t} \int_{0}^{s} \int_{0}^{1} e \int_{s}^{t} b(\eta) \mathrm{d} \eta\left[\frac{3 h^{2} \alpha_{1}}{\pi^{2}} T_{m}^{2} g_{1}^{2}\left(x_{1}, \eta\right)+\frac{3 h^{2} \alpha_{2}}{\pi^{2}} T_{m}^{2} g_{2}^{2}\left(x_{1}, \eta\right)+\beta\left(T^{*}\right)^{2}\left(x_{1}, \eta\right)\right] \mathrm{d} x_{1} \mathrm{~d} \eta \mathrm{d} s .
\end{aligned}
$$

It is obvious that $u \longrightarrow u^{*}, v \longrightarrow v^{*}$, and $T \longrightarrow T^{*}$ in $L_{2}(\Omega)$, as well as $H^{1}(\Omega)$, when $g_{1}, g_{2}, T^{*} \longrightarrow 0$ from inequality (96).

\section{Data Availability}

All data generated or analyzed during this study are included within the article.

\section{Conflicts of Interest}

The author declares that there are no conflicts of interest.

\section{Acknowledgments}

This work was supported by the Key Projects of Universities
in Guangdong
Province
(Natural Science)

\section{References}

[1] R. Lewandowski, Analyse Mathématique et Océanographie, Masson, Paris, France, 1997.

[2] A. Majda, Introduction to PDEs and Waves for the Atmosphere and Ocean, New York University, Courant Institute of Mathematical Sciences American Mathematical Society, New York, NY, USA, 2003.

[3] J. Pedlosky, Geophysical Fluid Dynamics, Springer, New York, NY, USA, 2nd edition, 1987.

[4] G. K. Vallis, Atmospheric and Oceanic Fluid Dynamics, Cambridge University Press, Cambridge, UK, 2006.

[5] W. M. Washington and C. L. Parkinson, An Introduction to Three Dimensional Climate Modeling, Oxford University Press, Oxford, UK, 1986.

[6] Q. C. Zeng, Mathematical and Physical Basis of Numerical Weather Prediction, Science Press, Beijing, China, 1979.

[7] J. L. Lions, R. Temam, and S. Wang, "New formulations of the primitive equations of atmosphere and applications," Nonlinearity, vol. 5, no. 2, pp. 237-288, 1992. 
[8] J.-L. Lions, R. Temam, and S. Wang, "On the equations of the large-scale ocean,” Nonlinearity, vol. 5, no. 5, pp. 1007-1053, 1999.

[9] J. Sun and S. Cui, "Sharp well-posedness and ill-posedness of the three-dimensional primitive equations of geophysics in Fourier-Besov spaces," Nonlinear Analysis: Real World Applications, vol. 48, pp. 445-465, 2019.

[10] M. Hieber, A. Hussein, and T. Kashiwabara, "Global strong $L^{p}$ well-posedness of the $3 \mathrm{D}$ primitive equations with heat and salinity diffusion," Journal of Differential Equations, vol. 261, no. 12, pp. 6950-6981, 2016.

[11] B. You and F. Li, "Global attractor of the three-dimensional primitive equations of large-scale ocean and atmosphere dynamics," Zeitschrift für Angewandte Mathematik und Physik, vol. 69, no. 5, pp. 1-13, 2018.

[12] E. Chiodaroli and M. Michálek, "Existence and nonuniqueness of global weak solutions to inviscid primitive and Boussinesq equations," Communications in Mathematical Physics, vol. 353, no. 3, pp. 1201-1216, 2017.

[13] J. Y. Sun and M. Yang, "Global well-posedness for the viscous primitive equations of geophysics," Boundary Value Problems, vol. 2016, no. 1, pp. 1-16, 2016.

[14] G. Boling and H. Daiwen, "On the 3D viscous primitive equations of the large-scale atmosphere," Acta Mathematica Scientia, vol. 29, no. 4, pp. 846-866, 2009.

[15] Y. Liu, "Continuous dependence for a thermal convection model with temperature-dependent solubility," Applied Mathematics and Computation, vol. 308, pp. 18-30, 2017.

[16] Y. Liu, S. Xiao, and Y. Lin, "Continuous dependence for the Brinkman-Forchheimer fluid interfacing with a Darcy fluid in a bounded domain," Mathematics and Computers in Simulation, vol. 150, pp. 66-82, 2018.

[17] Y. Liu, Y. Du, and C. Lin, "Convergence results for forchheimer's equations for fluid flow in porous media," Journal of Mathematical Fluid Mechanics, vol. 12, no. 4, pp. 576-593, 2010.

[18] N. L. Scott, "Continuous dependence on boundary reaction terms in a porous medium of Darcy type," Journal of Mathematical Analysis and Applications, vol. 399, no. 2, pp. 667-675, 2013.

[19] N. L. Scott and B. Straughan, "Continuous dependence on the reaction terms in porous convection with surface reactions," Quarterly of Applied Mathematics, vol. 71, no. 3, pp. 501-508, 2013.

[20] Y. Li and C. Lin, "Continuous dependence for the nonhomogeneous Brinkman-Forchheimer equations in a semiinfinite pipe," Applied Mathematics and Computation, vol. 244, pp. 201-208, 2014.

[21] A. A. Hameed and A. J. Harfash, "Continuous dependence of double diffusive convection in a porous medium with temperature-dependent density," Basrah Journal of Science, vol. 37, pp. 1-15, 2019.

[22] Y. F. Li, "Continuous dependence on the viscosity coefficient for the primitive equations," Journal of Shandong University (Natural Science), vol. 54, no. 12, pp. 12-23, 2019.

[23] Y. F. Li, "Continuous dependence on boundary parameters for three-dimensional viscous primitive equation of largescale ocean atmospheric dynamics," Journal of Jilin University (Science Edition), vol. 57, no. 5, pp. 1053-1059, 2019.

[24] M. Petcu, R. Temam, R. Temam, and D. Wirosoetisno, "Existence and regularity results for the primitive equations in two space dimensions," Communications on Pure \& Applied Analysis, vol. 3, no. 1, pp. 115-131, 2004.
[25] D.-W. Huang and B.-L. Guo, "On two-dimensional largescale primitive equations in oceanic dynamics (I)," Applied Mathematics and Mechanics, vol. 28, no. 5, pp. 581-592, 2007.

[26] C. Cao and E. Titi, "Global well-posedness of the three-dimensional viscous primitive equations of large scale ocean and atmosphere dynamics," Annals of Mathematics, vol. 166, no. 1, pp. 245-267, 2007.

[27] D.-W. Huang and B.-L. Guo, "On two-dimensional largescale primitive equations in oceanic dynamics (II)," Applied Mathematics and Mechanics, vol. 28, no. 5, pp. 593-600, 2007.

[28] C. H. Hardy, J. E. Littlewood, and G. Polya, Inequalities, Cambridge University Press, Cambridge, UK, 1953.

[29] D. S. Mitronovic, Analytical Inequalities, Springer-Verlag, Berlin, Germany, 1970. 